\title{
Sputter deposition processes
}

\author{
D. Depla $^{1}$, S. Mahieu ${ }^{1}$, J.E. Greene ${ }^{2}$ \\ ${ }^{1}$ Ghent University, Department of Solid State Sciences, Krijgslaan 281 (S1), 9000 \\ Ghent, Belgium \\ 2 Materials Science and Physics Departments and the Frederick Seitz Materials \\ Research Laboratory, University of Illinois, Urbana, Illinois 61801, USA
}

\begin{abstract}
Sputter deposition is a widely used technique to deposit thin films on substrates. The technique is based upon ion bombardment of a source material, the target. Ion bombardment results in a vapor due to a purely physical process, i.e. the sputtering of the target material. Hence, this technique is part of the class of physical vapor deposition techniques, which includes, for example, thermal evaporation and pulsed laser deposition. The most common approach for growing thin films by sputter deposition is the use of a magnetron source in which positive ions present in the plasma of a magnetically enhanced glow discharge bombard the target. This popular technique forms the focus of this chapter. The target can be powered in different ways, ranging from dc for conductive targets, to rf for nonconductive targets, to a variety of different ways of applying current and/or voltage pulses to the target. Since sputtering is a purely physical process, adding chemistry to, for example, deposit a compound layer must be done ad hoc through the addition of a reactive gas to the plasma, i.e. reactive sputtering. The undesirable reaction of the reactive gas with the target material results in a non-linear behavior of the deposition parameters as a function of the reactive gas flow. To model this behavior, the fluxes of the various species towards the target must be determined. However, equally important are the fluxes of species incident at the substrate because they not only influence the reactive sputter deposition process, but also control the growth of the desired film. Indeed, the microstructure of magnetron sputter deposited films is defined by the identity of the particles arriving at the substrate, their fluxes and the energy per particle.
\end{abstract}

\section{Introduction: how popular is sputter deposition?}

One way to compare sputter deposition with other deposition techniques, is to count the relative number of scientific publications and patents published each year for each deposition technique. In order to provide a baseline for the rate of increase in publications in general, we first determine the number of publications per year that refer to the combination of keywords "gold" OR "silver" OR "copper" on the Web of Sciences [1]. Similarly, a baseline for published patents is easily found by counting the number of patents published annually by entering the search term "the" in the Delphion Database [2]. Figure 1a is plot of the relative number of scientific papers per year per deposition technique. Figure $1 \mathrm{~b}$ provides the same information for patents. For the different physical vapor deposition techniques, magnetron sputtering is clearly used extensively in the scientific community, and competes with pulsed laser deposition (PLD) as the most important deposition technique. From the search in the patent database, it can be concluded that sputter deposition is still the most popular technique.

Hence, a chapter on sputter deposition in a book about thin films is quite relevant since this technique is applied in both research laboratories and industral plants to deposit a wide variety of materials. In this chapter, we focus on key aspects of sputter deposition. Describing the physics behind the sputter process; i.e. the interaction between the ion and the target, is a first priority. 
However, since many review articles are available [3-9], only the essential points are discussed here. Then, a basic system design is described. It should be noted that in this chapter we exclude ion beam sources, which have been well reviewed in the literature [10], due to limitations associated with scalability and power supply options. In the following sections several discharge sputter deposition configurations are discussed.

Sputter deposition is also used to deposit compound films by adding a reactive gas to the discharge. This, however, greatly increases the complexity of the deposition process, and explains the ongoing interest in academia to investigate this technique for which several aspects are not completely understood.

Sputtered target atoms are ejected with substantial kinetic energy, of the order of or larger than bond energies, and hence can significantly affect film growth kinetics and microstructure. Thus, energy loss mechanisms during transport in the gas phase are important. The chapter ends with a discussion of the typical microstructure of sputter deposited coatings.

\section{What is sputtering ?}

Sputtering is the ejection of atoms by the bombardment of a solid or liquid target by energetic particles, mostly ions. It results from collisions between the incident energetic particles, and/or resultant recoil atoms, with surface atoms. A measure of the removal rate of surface atoms is the sputter yield Y, defined as the ratio between the number of sputter ejected atoms and the number of incident projectiles. Excellent review articles on sputtering are available in the literature [3-9], and only the essential features are discussed here.

Based on the large amount of experimental (see e.g. [11]) and calculated data as a function of ion and target material, several trends are apparent. For a given ion mass and target, Y exhibits a maximum as a function of ion energy as well as a minimum (threshold) energy. An example is shown in figure 2 for $\mathrm{Ar}^{+}$bombardment of $\mathrm{Cu}$.

When comparing the sputter yield of target materials bombarded by a given ion at constant energy, one notices a trend related to the position of the element in the periodic table (see figure 3 and the following discussion).

Several authors have derived equations describing the sputter yield as a function of energy and projectile-target combinations. P. Sigmund is the father of these theories. His work "Theory of Sputtering I. Sputter yield of Amorphous and Polycrystalline targets" published in Physical Review [12] is a benchmark in this field. According to the theory of Sigmund, the sputter yield near threshold, i.e. at low ion energy, is given by

$$
Y=\frac{3}{4 \pi^{2}} \alpha \frac{4 M_{1} M_{2}}{\left(M_{1}+M_{2}\right)^{2}} \frac{E}{U_{s}}
$$

with $\mathrm{E}$ the energy of the projectile and $\mathrm{M}_{1}$ and $\mathrm{M}_{2}$ the masses of the projectile and the target atom (in $\mathrm{amu}$ ). $\mathrm{U}_{\mathrm{s}}$ is the surface binding energy and $\alpha$ a dimensionless parameter depending on the mass ratio and the ion energy. At low energy, and mass ratios $\mathrm{M}_{2} / \mathrm{M}_{1}$ lower than $1, \alpha$ is of the order of 0.2. This equation can be understood as follows. An incoming ion transfers its momentum to the target atoms which explains the term $4 M_{1} M_{2} /\left(M_{1}+M_{2}\right)^{2}$ with a maximum when $M_{1}=M_{2}$. To sputter an atom from the target, momentum transfer from the ion-induced collision must overcome the surface barrier, given by the surface binding energy $U_{s}$. Therefore, we can expect an inversely proportional relationship between the yield and the surface binding energy. Based on equation (1), we can expect that for the energy range of interest for sputter deposition, the sputter yield will vary linearly with the ion energy (see figure 2). The behavior of the sputter yield over the periodic table can also be understood from equation (1), because the sputter yield is defined by momentum transfer and surface binding energy. However, differences in atomic density among different materials also affect $\mathrm{Y}$ through variations in the range (depth) of momentum transfer.

In addition to the theory of Sigmund, heuristic approaches based on semi-empirical equations, and simulations (for an overview on sputter yield simulations, see [13]) are also available. 
Commonly used semi-empirical formulae for the calculation of the sputter yield were developed by Yamamura et al. [14]. The equations are valid for the bombardment of monoatomic solids by projectiles at normal incident, and the sputter yield $\mathrm{Y}(\mathrm{E})$ is given by

$$
Y(E)=0.042 \frac{Q\left(Z_{2}\right) \alpha\left(M_{2} / M_{1}\right)}{U_{s}} \frac{S_{n}(E)}{1+\Gamma k \varepsilon^{0.3}}\left[1-\sqrt{\frac{E_{\text {th }}}{E}}\right]^{s}
$$

with $E, M_{1}, M_{2}$ and $U_{s}$ as defined for the equation (1). $U_{s}$, the surface binding energy, is intimately connected with, and explains the presence of the threshold energy $\left(\mathrm{E}_{\mathrm{th}}\right)$ for sputtering (see table I). The other symbols are defined in table I.

Although these equations provide a value for the sputter yield $\mathrm{Y}$ as a function of ion energy and material choice, they are not instructive in explaining the sputtering process in detail.

\begin{tabular}{|c|c|c|}
\hline Symbol & Definition & \\
\hline $\mathrm{Q}\left(\mathrm{Z}_{2}\right)$ & tabulated dimensionless parameter & \\
\hline$\alpha$ & $0.249\left(\mathrm{M}_{2} / \mathrm{M}_{1}\right)^{0.56}+0.0035\left(\mathrm{M}_{2} / \mathrm{M}_{1}\right)^{1.5}$ & $\mathrm{M}_{1} \leq \mathrm{M}_{2}$ \\
\hline \multirow{3}{*}{$\mathrm{E}_{\text {th }}$} & $0.0875\left(\mathrm{M}_{2} / \mathrm{M}_{1}\right)^{-0.15}+0.165\left(\mathrm{M}_{2} / \mathrm{M}_{1}\right)$ & $M_{1} \geq M_{2}$ \\
\hline & $\frac{6.7}{\gamma} \mathrm{U}_{\mathrm{s}}$ & \\
\hline & $\frac{1+5.7\left(\mathrm{M}_{1} / \mathrm{M}_{2}\right)}{\gamma} \mathrm{U}_{\mathrm{s}} \quad \mathrm{M}_{1} \leq \mathrm{M}_{2}$ & \\
\hline$\gamma$ & $\frac{4 M_{1} M_{2}}{\left(M_{1}+M_{2}\right)^{2}}$ & \\
\hline$\Gamma$ & $\mathrm{W}\left(\mathrm{Z}_{2}\right)$ & \\
\hline & $\overline{1+\left(M_{1} / 7\right)^{3}}$ & \\
\hline $\mathrm{W}\left(\mathrm{Z}_{2}\right)$ & tabulated dimensionless parameter & \\
\hline $\mathrm{s}$ & tabulated dimensionless parameter & \\
\hline $\mathrm{k}$ & The Linhard electronic stopping coefficient & \\
\hline $\mathrm{S}_{\mathrm{n}}(\mathrm{E})$ & $\frac{84.78 Z_{1} Z_{2}}{\left(Z_{1}^{2 / 3}+Z_{2}^{2 / 3}\right)^{1 / 2}} \frac{M_{1}}{M_{1}+M_{2}} s_{n}^{T F}(\varepsilon)$ & \\
\hline$\varepsilon$ & Lindhard-Scharff-Schiott reduced energy & \\
\hline $\mathrm{S}_{\mathrm{n}}^{\mathrm{TF}}(\varepsilon)$ & The reduced nuclear stopping cross section & \\
\hline
\end{tabular}

Table I. Overview of the terms in Yamamura formulae. For more details, see [14].

Hence, some authors have developed simpler, and more transparent, models of the sputtering process. An excellent example is the work of Mahan et al. [15] in which the sputter yield Y(E) is derived based on the following assumptions. The effective number of recoiling target atoms created per incident ion is multiplied by the probability that the recoil is close enough to the surface to escape and by the probability that the recoils are travelling towards the surface, or

$$
\mathrm{Y}=\frac{\mathrm{E}}{\mathrm{E}_{\mathrm{avg}}} \frac{\mathrm{R}_{\mathrm{pr}}}{\mathrm{R}_{\mathrm{pp}}} \frac{1}{4}
$$

with $E$ the projectile energy and $E_{a v g}$ the average energy of the recoils. The ratio $E / E_{\text {avg }}$ gives the average number of recoils. The ratio between the projected range of the recoils $R_{p r}$ and the projected range of the projectile $R_{p p}$ gives the probability that the recoils are close enough to the surface to escape. Finally the term $1 / 4$ is the average probability that the recoils are moving towards the surface. Using approximations, the average recoil energy and the projected range can be calculated straightforwardly giving insight into the sputtering process. A good example of this is the 
calculation of the threshold energy. The physics behind the threshold energy is that the recoil atom has insufficient energy to overcome the surface energy barrier $U_{s}$ when its average energy $E_{\text {avg }}$ is equal to or lower than the surface barrier energy. The average recoil energy in this simplified model is calculated as

$$
\mathrm{E}_{\mathrm{avg}}=\mathrm{U}_{\mathrm{s}} \ln \left(\gamma \mathrm{E} / \mathrm{U}_{\mathrm{s}}\right)
$$

and the threshold energy $E=E_{\text {th }}$ is therefore found by substituting $U_{s}$ for $E_{\text {avg, }}$, and obtaining $\mathrm{E}_{\mathrm{th}}=2.72 \mathrm{U}_{\mathrm{s}} / \gamma$, which is similar to the formulae proposed by Yamamura et al. (see Table I).

Another approach to obtain a value for the sputter yield is to simulate the overall sputtering process (see [13] for an overview). The most commonly used simulation package is the well known SRIM code [16] developed by Ziegler et al. and completely described in their book [17]. Figures 2 and 3 were simulated using this code. All features discussed in the previous paragraphs are present in both figures. SRIM is a static simulation code. That is, it does not account for target changes due to the ion bombardment itself. To account for these changes, Möller et al. developed a dynamic version of SRIM, i.e. TRIDYN [18].

All approaches also provide the angular and energy distribution of the sputtered particles. Thompson [19] showed that within a certain (low) ion energy range, the energy distribution follows the expression

$$
\frac{d Y}{d E} \propto \frac{E}{\left(E+U_{s}\right)^{3}},
$$

which is based on the assumption of a planar surface barrier for sputtered particles. This expression gives a peak at $U_{\mathrm{s}} / 2$. Falcone[20] estimated the average energy of sputtered particles $\overline{\mathrm{E}}$ as

$$
\bar{E}=2 U_{s}\left(\ln \frac{E}{E_{\text {th }}}-\frac{3}{2}\right)
$$

Substituting reasonable values into equation (6) shows that the energy of the sputtered particles is at least one order of magnitude higher than the corresponding thermal evaporation energy for the same particle flux (see Figure 4). Indeed, at $1000 \mathrm{~K}$ the thermal energy is only of the order of $0.1 \mathrm{eV}$. With a surface binding energy of a few $\mathrm{eV}$ (for $\mathrm{Cu}$ it is $3.5 \mathrm{eV}$ [17]), the maximum in the energy distribution occurs at $1.8 \mathrm{eV}$, and using the SRIM calculated threshold energy, the average energy is $15.1 \mathrm{eV}$. If the pressure during deposition is low enough, the sputtered particles in the gas phase are ballistic and can reach the substrate without few or no collisions in the gas phase.

In all of this work, one important concept is not addressed at all, i.e. the sputter yield of compound materials. The Yamamura formulae can be applied to multicomponent materials such as compounds and alloys using weighted average values for $Z_{2}, M_{2}$, and $U_{s}$ [13]. Surface binding energies, in particular, are difficult to obtain for oxides and nitrides. For metals, the surface binding energy is generally set equal to the vaporization enthalpy, but this approach is not applicable to oxides, nitrides, sulphides, etc. In the context of the preferential sputtering of oxygen from oxides during depth profiling of oxide thin films for analytical approaches, models have been proposed to estimate a value of the surface binding energy of the metal and the oxygen atoms. Using these values, the modification of the surface composition by ion bombardment is calculated and compared with experimental values. Results typical for this kind of study, including a model for the surface binding energy, are published by Malherbe et al.[21]. Some simulation codes, e.g. TRIDYN, use a different approach [18] where the effective surface binding energy of $\mathrm{O}$ and $\mathrm{M}$ are chosen to be dependent on the actual surface composition by use of a matrix method. The matrix elements of surface binding energies are $\mathrm{SBV}_{\mathrm{O}-\mathrm{O}}, \mathrm{SBV}_{\mathrm{O}-\mathrm{M}}, \mathrm{SBV}_{\mathrm{M}-\mathrm{O}}$ and $\mathrm{SBV}_{\mathrm{M}-\mathrm{M}}$. These elements are evaluated by the formulae

$$
\begin{aligned}
& \mathrm{SBV}_{\mathrm{O}-\mathrm{O}}=0 \\
& \mathrm{SBV}_{\mathrm{M}-\mathrm{M}}=\mathrm{U}_{\mathrm{s}, \mathrm{M}} \\
& \mathrm{SBV}_{\mathrm{O}-\mathrm{Al}}=\frac{1}{2} \mathrm{U}_{\mathrm{s}, \mathrm{M}}+\frac{\mathrm{n}+\mathrm{m}}{2 \mathrm{~nm}} \Delta \mathrm{H}^{\mathrm{f}}+\frac{\mathrm{n}+\mathrm{m}}{4 \mathrm{n}} \Delta \mathrm{H}^{\text {diss }}
\end{aligned}
$$


where $n$ and $m$ depend on the stoichiometry of the oxide $\mathrm{M}_{\mathrm{n}} \mathrm{O}_{\mathrm{m}}$. $\mathrm{U}_{\mathrm{s}, \mathrm{M}}$ is the metal surface binding energy. $\Delta \mathrm{H}^{\mathrm{f}}$ denotes the formation enthalpy per molecule of the compound and $\Delta \mathrm{H}^{\text {diss }}$ denotes the dissociation energy of the oxygen molecule. If the concentrations of $\mathrm{O}$ and $\mathrm{M}$ at the surface are $\mathrm{C}_{\mathrm{O}}$ and $\mathrm{C}_{\mathrm{M}}$, respectively, we obtain the surface binding energy of $\mathrm{M}$ and $\mathrm{O}$ in the following way.

$$
\begin{aligned}
& \operatorname{SBE}(M)=C_{O} \cdot \operatorname{SBV}_{\mathrm{O}-M}+C_{M} \cdot \operatorname{SBV}_{M-M} \\
& \operatorname{SBE}(O)=C_{M} \cdot S^{S B V} V_{O-M}+C_{O} \cdot S^{S B V} V_{O-O}
\end{aligned}
$$

\section{How to generate the energetic particles?}

Sputtering is initiated due to the bombardment of energetic particles at the target. These energetic particles are generally ions. Two approaches can be followed to produce ions and sputter the target materials. The first is quite straightforward by using an ion source which is aimed toward the target. Collecting the sputtered particles on a substrate enables the deposition of a thin film. However, ion beam sputtering is not widely used for industrial large scale applications. Ions guns are more often utilized in surface analytical techniques such as SIMS (secondary ion mass spectrometry) or to bombard the substrate during thin film deposition[22]. As such, these external sources of ions will not be covered in this chapter. A good overview can be found in [10].

Another source of ions is a plasma. By applying a high negative voltage to the cathode, i.e. the target, positively charged ions are attracted from the plasma towards the target. The ions gain energy in the electric field and bombard the target with sufficient energy to initiate sputtering. Sputtering was first discussed in the literature by W.R. Grove in 1852 who used this kind of set-up [23].

A good starting point to discuss plasma based sputter deposition is using the simplest experimental arrangement. That is, a cathode and an anode are positioned opposed to each other in a vacuum chamber. Typically, the vacuum chamber is pumped by a combination of turbomolecular and rotary pumps, although a diffusion pump is still often used. After pumping to a base pressure of the order of $1 \times 10^{-4} \mathrm{~Pa}^{1}$ or lower, a noble gas (usually argon) is introduced into the vacuum chamber reaching a pressure between 1 to $10 \mathrm{~Pa}$. When a high voltage in the range of $2000 \mathrm{~V}$ is applied between cathode and anode, a glow discharge is ignited. It is not in the scope of this chapter on sputter deposition to describe all details related to a glow discharge, but discussing a few can be instructive. To define a glow discharge, one can follow the current-voltage (I-V) characteristic. It is important to realize that these characteristics depend also on the pressure and the separation between cathode and anode. The main characteristics of the discharge, such as breakdown voltage, I-V characteristic, and structure of the discharge, depend on the geometry of the electrodes (cathode and anode) and vacuum vessel, the gas(es) used and the electrode material. The I-V characteristics of such a discharge are illustrated in Figure 5 for a wide range of currents. Three general regions can be identified in the figure, the dark discharge region, the glow discharge and, the arc discharge. The electric circuit of the discharge gap also includes an external ohmic resistance R. In this case, Ohm's law for the circuit can be written as

$\mathrm{EMF}=\mathrm{V}+\mathrm{RI}$

where EMF is the electromotive force and $\mathrm{V}$ is the voltage of the gas discharge. Equation (8) is usually referred to as the load line, and is also shown in Figure 5. Intersection of the I-V characteristic and the load line gives the actual value of current and voltage in a discharge. By adjusting the ballast resistor in the circuit diagram, we can sweep out an I-V characteristic that is highly nonlinear and shows the three general regions. Each of these regions encompasses many interesting phenomena.

\footnotetext{
${ }^{1}$ Other commonly used pressure units are mTorr and mbar. 1 mTorr corresponds to $0.133 \mathrm{~Pa}$. 1 mbar corresponds to $100 \mathrm{~Pa}$.
} 
Let us first focus on the dark discharge regime, between $\mathrm{A}$ and $\mathrm{E}$ in figure 5 . The name refers to the fact that the discharge remains invisible to the eye, i.e. there is no visible light emitted except for the corona discharge and the breakdown itself. The change in the I-V characteristic can be understood from a description of the responsible physical processes. Between A and B, the ions and electrons formed by the background ionization move towards the electrodes due to the applied electrical field, producing a weak electrical current. Increasing the applied voltage results in a better collection efficiency, i.e. a larger fraction of the produced ions and electrons will reach the electrodes. At a sufficient high voltage, the current will saturate because all produced electrons and ions reach the electrodes. Hence, in the region between $\mathrm{B}$ and $\mathrm{C}$, the current remains constant with increasing voltage. Some radiation counters, e.g. a Geiger-Müller counter, make use of the fact that the measured current will depend linearly on the strength of the radiation source. When the voltage across the low pressure discharge tube is increased further, one notices a strong increase in current (see regions $\mathrm{C}$ to $\mathrm{E}$ ). Hence, more electrons and ions must be produced. The origin of the current increase is found in the impact ionization of atoms by the original electrons accelerated across the electric field. Hence, an avalanche of electron and ion production will follow leading to a strong increase in current. This region is called the Townsend discharge.

Corona discharges (D to E) occur in Townsend dark discharges, prior to electrical breakdown, in regions of high electric field near sharp points, edges, or wires. If the corona currents are high enough, corona discharges are technically "glow discharges" and visible to the eye. For low currents, the entire corona is dark. Corona discharges are often applied to treat the surface of polymers and to render the surface more "active" by breaking atomic bonds. Finally, at sufficiently high electrical fields, breakdown will occur due to addition of secondary electrons emitted from the cathode as a result of ion and photon impact (see below). At the breakdown potential (point E), the current may increase significantly, and is usually limited by the internal resistance of the power supply connected between the plates. If the internal resistance of the power supply is very high, the discharge tube cannot draw enough current to break down the gas, and the tube will remain in the corona regime with small corona points (brush discharges) present on the electrodes. If the internal resistance is lower, then the gas will break down at the indicated voltage and move into the normal discharge regime (region F-G). The breakdown voltage for a particular gas and electrode material depends on the product of the pressure $\mathrm{P}$ and the distance $\mathrm{d}$ between the electrodes, as expressed in Paschen's law,

$$
\mathrm{V}_{\text {Breakdown }}=\frac{\mathrm{BPd}}{\ln (\mathrm{APd})+\ln \left(\ln \left[\frac{1}{\gamma}+1\right]\right)}
$$

with $\mathrm{A}$ and $\mathrm{B}$ constants, and $\gamma$ the electron emission yield induced by photon and ion bombardment. The constants A and B depend on the chosen gas and define the Townsend ionization coefficient. This latter coefficient gives the electron production per unit length, or the multiplication of the electrons per unit length along the electric field.

In contrast to the dark discharge regime, the plasma in the region F-G is luminous in the visible, and hence it is called a glow discharge. The excitation of the gas atoms by electron impact forms the origin of the gas glow. The plasma density is now sufficiently high that the electric field between the electrodes becomes distorted from its original configuration in the Townsend discharge. Along the discharge, one can notice, especially at low pressure, a sequence of dark and bright layers. These layers have special names. Close to the cathode is a dark layer known as the Aston dark space followed by a thin layer of the cathode glow. This bright layer is then followed by the cathode dark space. Sharply separated from this latter region is the negative glow. The luminosity of the negative glow decreases toward the anode, becoming the Faraday dark space. After the Faraday dark space is the positive column. At the anode side, the positive column goes over into the anode dark space followed by a narrow anode glow. A schematic overview is shown in figure 6 . 
Most of the voltage drop between cathode and anode occurs between the cathode and the negative glow. The length of the cathode fall region or "the dark space' from the cathode to the boundary of the negative glow is typically a few centimeters. So, within this region most power is dissipated and one notices a strong voltage drop. The voltage drops over a distance which is generally not exactly equal to the width of the dark space. To distinguish between the two regions, one refers to the first region as the cathode sheath. When the glow discharge covers only a part of the cathode, the discharge is in the normal glow discharge mode. In this regime, the current density at the electrodes is independent of the discharge voltage and hence by increasing the current, the part covered by the plasma increases at constant discharge voltage (see region F-G). From point G on, the plasma completely covers the cathode surface and with increasing discharge current, the discharge voltage increases. In this regime, the abnormal discharge region, sputter deposition is typically performed. At point $\mathrm{H}$, the electrodes become sufficiently hot that the cathode now emits electrons thermionically. If the dc power supply has a sufficiently low internal resistance, the discharge will undergo a transition from glow to arc. As the energy of the arriving fast neutrals and ions defines the sputter yield, it is interesting to study the typical ion energy distribution in a glow discharge. Indeed, the erosion speed of the target will be defined by the flux of ions and fast neutrals bombarding the cathode and the sputter yield which depends on the energy and mass of these species. At the typical pressures of a dc argon glow discharge, the $\mathrm{Ar}^{+}$ions have a small mean free path. This means that the distance the ion travels before it makes a collision, or it ceases to be an ion, is short. The short mean free path is due primarily to the following effect. When an $\mathrm{Ar}^{+}$ion passes close enough (a few $\AA$ ) to an Ar atom, quantum mechanical tunneling occurs. So, the ion becomes again an atom, while the atom from which the electron is removed now becomes an ion. This process is called a symmetric charge exchange collision because the result is still an ion and an atom. However, the ion which has already gained energy by acceleration in the electric field within the cathode region becomes a neutral Ar atom with the same energy, while the atom becomes an ion with the low energy of the atom. This newly created ion is now accelerated by the voltage gradient in the cathode region. The fast neutral Ar atom can not gain energy anymore, and due to the high pressure will lose energy by collision with other atoms. So, even with the strong electric field, defined by the high discharge voltage and the cathode sheath thickness, the number of high energy ions arriving at the cathode will be quite small. Davis and Vanderslice [24] fit measured the energy distributions of the ions reaching the target based on the following equation:

$\frac{\mathrm{V}_{\mathrm{C}}}{\mathrm{N}_{0}} \frac{\mathrm{dN}}{\mathrm{dV}}=\left(\frac{\mathrm{L}}{\lambda}\right)\left[\frac{1}{2\left(1-\mathrm{V}_{\mathrm{x}} / \mathrm{V}_{\mathrm{C}}\right)^{1 / 2}}\right] \mathrm{e}^{-(\mathrm{L} / \lambda)\left[1-\left(1-\mathrm{V}_{\mathrm{x}} / \mathrm{V}_{\mathrm{c}}\right)^{1 / 2}\right]}$,

with $\mathrm{N}_{\mathrm{o}}$ the number of ions starting from the negative glow, $\mathrm{L}$ the sheath thickness and $\lambda$ the mean free path for charge transfer.

This distribution is plotted for various values of $\mathrm{L} / \lambda$ in figure 7 . It will be noted that the ions that do not suffer any collisions, but arrive at the cathode with the full cathode fall energy have not been included so far. Their relative number, given by $\mathrm{e}^{-\mathrm{L} / \lambda}$, is indicated at the right end of the curves. So, with an experimental value of $L / \lambda$ of 25 (see Davis and Vanderslice [24]), we can conclude that essentially no ions arrive at the target with energy equal to the applied target voltage. Experiments and simulation on this topic are also described by Bogaerts et al. [25][26].

While the sputter yield is determined by the energy and mass of the ions at the target, the sputtering rate of the target depends on the total power. For a simple de diode system, the ion current density is uniform over nearly the entire target during the abnormal glow discharge mode. Experimentally, one finds that the current density is proportional to $\left(\mathrm{V}-\mathrm{V}_{0}\right)^{3 / 2}$ where $\mathrm{V}_{0}$ is the voltage required to maintain the discharge, and to $\left(\mathrm{p}-\mathrm{p}_{0}\right)$ where $\mathrm{p}_{0}$ is the gas pressure needed to maintain the discharge. So, to obtain higher current densities it is necessary to increase either $\mathrm{V}$ or $\mathrm{p}$, or both. A high pressure is not interesting as the energy of the sputtered particles arriving at the substrate is low and many sputtered atoms are scattered back to the target. Increasing the discharge voltage is also not an option. Many electrons that are ejected from the target will reach the anode without losing much 
energy in collisions because the ionization cross section decreases strongly with increasing electron energy. Hence, when the electron has obtained its full energy by acceleration in the electrical field in the cathode region, it is less likely to ionize gas atoms far from the cathode. Increasing the discharge voltage results in a higher electron current density at the anode. These energetic electrons can deliver significant power to the anode, thus resulting in substrate heating which may limit the substrate choice for this deposition process.

In conclusion, dc glow discharge sputtering has some drawbacks as a deposition process. Hence, several other sputtering techniques have been developed to overcome these drawbacks. The most important technique is magnetron sputter deposition which is the subject of the next section.

\section{Efficient trapping of electrons leads to magnetron sputter deposition}

In a diode glow discharge arrangement, electron trajectories are only defined by the electrical field between the cathode and the anode. Hence, the electrons are accelerated over the cathode sheath, and move with high velocity towards the anode. To be sure the electrons produces sufficient ions to sustain the discharge, the pressure must be quite high, i.e. of the order of a few Pa. The classical approach to avoid the rapid loss of electrons from the discharge is to apply a magnetic field. By applying a magnetic field during glow discharge sputter deposition, one can trap the electrons in the discharge longer and, hence, produce more ions for the same electron density. As the electron trajectory is elongated, the probability of ionizing a gas atom during their travel from cathode to anode increases, which enables a reduction in the discharge pressure and the cathode sheath. In this way, the ions can reach the cathode with almost the full discharge voltage and the sputtered atoms can reach the substrate with only a few collisions. Also, the deposition rate, a technically meaningful criterion, will dramatically increase compared to simple diode glow discharge systems.

\subsection{Post magnetrons}

In post magnetrons a uniform magnetic field, $\vec{B}$, is applied parallel to the surface of a cylindrical target. It has approximately the same strength at all points on the target surface. Electrons emitted from the target by ion impact are accelerated by the potential over the sheath (see section 3 ). The presence of the magnetic field influences the trajectory of the electrons, and forces them in cycloidal orbits, bringing them back to the cathode, unless they make a collision. The electron trajectory can be described by the Lorenz equation,

$$
\vec{F}=q(\vec{E}+\vec{V} \times \vec{B}) \text {, }
$$

with q the negative charge of the electron, $\vec{E}$ the electric field over the dark space, $\vec{B}$ the magnetic field, and $\vec{v}$ the velocity of the electron. Once the electron has left the cathode sheath, its trajectory is defined primarly by the magnetic field. The Lorenz force on the electron depends on its velocity and the magnetic field strength, and is orthogonal to both their directions. The first component of the electron motion is their movement along the magnetic field lines. A second component is the gyration of the electrons around the magnetic field lines with the Larmor radius,

$r_{\mathrm{L}}=\frac{\mathrm{mv}}{\mathrm{qB}}$,

in which $\mathrm{v}_{\perp}$ is the velocity of the electron perpendicular to the target. Finally, the third component of the electron trajectory is the $\vec{E} \times \vec{B}$ Hall drift. This drifting "around" the post in a helical motion occurs perpendicular to the electric and magnetic fields. Summarizing, the electrons move continuously in one direction around the target and are accelerated and decelerated close to the target surface.

The average electron energy loss per ionization, $\mathrm{W}$, is of the order of $30 \mathrm{eV}$ for Ar magnetron discharges[27]. This number can be retrieved based on MC simulations (see e.g. [28]). If we assume that the voltage drop across the sheath is approximately equal to the discharge voltage $\mathrm{V}_{\mathrm{d}}, \mathrm{eV}_{\mathrm{d}} / \mathrm{W}$ ion-electron pairs will be produced on average by one electron. This implicitly assumes, as a first approximation, that there is no ionization in the sheath. This approximation is reasonable as the 
sheath width is of the order of a few millimeters (and varies as $1 / \mathrm{P}$ ), and much smaller than the electron path length. If all $\mathrm{eV}_{\mathrm{d}} / \mathrm{W}$ ions reach the cathode, and $\gamma_{\mathrm{e}}$ is the ion-induced electron emission yield, then $\left(\mathrm{eV}_{\mathrm{d}} / \mathrm{W}\right) \gamma_{\mathrm{e}}$ electrons will be emitted from the target. To sustain the discharge, we can write

$$
\frac{\mathrm{eV}}{\mathrm{W}} \gamma_{\mathrm{e}}=1
$$

from which we can derive an equation for the minimum required voltage,

$$
\mathrm{V}_{\mathrm{d}}=\frac{\mathrm{W}}{\mathrm{e} \gamma_{\mathrm{e}}} \text {. }
$$

Using a typical value for $\mathrm{W}$ of $30 \mathrm{eV}$, and $\gamma_{\mathrm{e}}$ of the order of 0.1 for metal targets (see e.g. [29]), a value of $-300 \mathrm{~V}$ is obtained. Of course, electrons can be lost to ground before they have created the maximum number of electron-ions pairs. A possible loss mechanism occurs when the magnetic field line which the electron follows crosses the anode or the chamber walls. Also, ions produced far from the cathode sheath will not reach the target. Both loss mechanisms can be included in equation (14) by introducing the coefficients $\varepsilon_{\mathrm{i}}$ and $\varepsilon_{\mathrm{e}}$ for ion and electron loss, respectively. The values of these coefficients are close to one for magnetrons. Thus,

$$
\mathrm{V}_{\mathrm{T}}=\frac{\mathrm{W}}{\mathrm{e} \gamma_{\mathrm{e}} \varepsilon_{\mathrm{i}} \varepsilon_{\mathrm{e}}}
$$

In the derivation of equation (15), we assumed that no ionization occurs in the sheath. However, when the magnetic field is strong, the electron paths are close to the target, and therefore substantial ionization can occur in the sheath. The electrons produced in the sheath will also gain energy and can contribute to the production of electron-ion pairs. This process can be seen as a multiplicative process for the original electron. We include this effect in equation (15) by introducing the multiplication parameter $\mathrm{m}$. We notice, as expected, that more sheath ionization results in a lower discharge voltage to sustain the discharge, i.e.

$$
\mathrm{V}_{\mathrm{T}}=\frac{\mathrm{W}}{\mathrm{e} \gamma_{\mathrm{e}} \varepsilon_{\mathrm{i}} \varepsilon_{\mathrm{e}} \mathrm{m}} \text {. }
$$

We can further improve equation (16) by realizing that an electron which doesn't interact with the gas will return to the target with its initial energy (typical a few eV [30]). Hence, an electron which has not collided with a gas atom, can be reflected at the target surface and continue its path, or it can be absorbed (or recaptured) by the target. In the latter case, the electron is lost for further ionization. Increased recapture will therefore result in a higher discharge voltage. To account for this effect we introduce the effective ionization probability $\mathrm{f}$ in equation (16) which is a number between 0 (all electrons are recaptured) and 1 (no electrons are recaptured):

$$
\mathrm{V}_{\mathrm{T}}=\frac{\mathrm{W}}{\mathrm{e} \gamma_{\mathrm{e}} \varepsilon_{\mathrm{i}} \varepsilon_{\mathrm{e}} \mathrm{mf}} \text {. }
$$

The pressure dependence of a magnetron discharge can be understood from this latter process. When the pressure in a dc diode glow discharge is reduced, the electrons emitted from the target will reach the anode without ionizing gas atoms, and hence the electron is lost for the ionization process. Therefore, at too low of a pressure, the dc diode glow discharge will extinguish. However, for a magnetron discharge, the high energy electron can not reach the anode very easily due to the presence of the magnetic field. Nevertheless, when the pressure is decreased, the magnetron discharge will ultimately extinguish. The loss process for the electrons is now electron recapture at the target. At high pressure, the effective ionization probability will be close to one because the emitted electrons can easily interact with the gas atoms. Inelastic collisions (such as ionization and excitation of the gas atoms) result in energy loss. Due to this energy loss, the electrons cannot return to the target. At lower pressure the ionization probability is smaller and hence electrons can be 
recaptured. Therefore, we expect a higher discharge voltage (when keeping the other parameters such as magnetic field, magnetron type and discharge current constant) at lower pressure.

In a post magnetron, flanges are placed at the end of the target. The flanges are at the same negative potential as the target, and prevent the electrons which drift over the target from escaping axially.

\subsection{Planar magnetrons}

Although cylindrical and planar magnetrons have different geometries, the basic principles of operation are the same, but are generally easier to understand from the symmetry of the cylindrical case. However, planar magnetrons are more widely used because of their convenience. One example is the easy ability to design air-to-air systems for flat substrates. In a planar magnetron design, permanent magnets are generally placed behind the target. For a circular target, there is a central disc magnetic pole and an annular pole so that the magnetic field lines between the poles have a circular symmetry. This way of generating a magnetic field has its consequences. First the magnetic field strength varies over the target surface. Also, the direction changes over the target surface as the direction of $\vec{B}$ is tangent to the field line. Thus, the magnetic field is only parallel to the target surface at unique points, but at most points the magnetic field has a component parallel to the target. The effect of this parallel component is similar to the one described in the previous section. It results in cycloid orbits above the target surface. The vertical component of the magnetic field is also important, preventing electrons from escaping the target by generating an electromagnetic "bottle". Indeed, as an electron moves along the target, it has its velocity vector parallel to the target surface. Hence, the presence of the vertical component of the magnetic field will force the electron (see equation 10) toward the central area between the magnets. For a circular

planar magnetron the $\vec{E} \times \vec{B}$ drift will be parallel to the target and the electron will follow a circular path around the target and will not escape the magnetic "bottle" (see figure 8) unless it collides with gas atoms.

In the case of a rectangular planar magnetron, the magnet configuration is designed to assist the electrons to move around corners of the target. For both circular and rectangular magnetrons, the magnet configuration results in maximum ionization in the region between the magnets. Hence, most ions will be formed at this position, and as the ions move in a perpendicular trajectory towards the target, the ion current density will be the highest at this position. For a circular magnetron, a torus-shaped plasma will be formed (see figure 9), leading to a circular "race track" erosion pattern. The sputtering process must be interrupted to prevent cooling water entering the vacuum system when the erosion groove becomes too deep such that it may break through the target. Moreover, the formation of the erosion pattern results in a continuously stronger magnetic field strength above the surface because the target surface recedes towards the magnets as material is sputtered. Hence, the erosion rate increases (and the voltage usually decreases) as a function of sputtering time. Several companies offer solutions to increase the target utilization. Optimization of the magnetic field design is a common solution, but even with an optimized magnet design target utilization remains less than $50 \%$ for stationary magnets. By moving the magnets in a complex geometry under the target, higher target utilization can be achieved.

\subsection{Rotating cylindrical magnetrons}

To improve target utilization, rotating cylindrical magnetrons have been developed. Their magnetic field design is quite similar to that of planar magnetrons. However, the target is a cylindrical tube and is rotated around a stationary magnet configuration. The disadvantage of these magnetrons is the same as for post magnetrons, i.e. a complex target design and fabrication. For some metals, a tube can be used, but for many materials the target material must be flame or plasma sprayed onto a cylinder or backing tube, which is water cooled and holds the magnet configuration. The bearing design is crucial with this magnetron type as the water flow is inside the vacuum system. The much higher target utilization, up to $85 \%$, is the main advantage. A major problem with planar targets is the built-up of compound material on the target during reactive magnetron sputtering. The compound layer on non-sputtered target parts forms the origin of arcing in insulating reactive 
systems such as $\mathrm{Al} / \mathrm{O}_{2}$. With a rotating cylindrical magnetron, this compound layer is sputtered again after each target rotation, resulting in better process stability during reactive magnetron sputter deposition.

\subsection{Some general features of magnetrons and magnetron discharges}

\subsubsection{The discharge voltage}

The discharge voltage is an easily measurable quantity and equation (17) helps to explain its behavior as a function of experimental parameters. We discuss the most important of these, and refer to [31] for more details.

When using a planar magnetron, the discharge voltage will decrease as a function of time. This behavior can be readily explained by the increase in magnetic field strength adjacent to the target (see section 4.2) due to the erosion groove formation. As such, electrons will move closer to the target, and more sheath ionization will occur, and according to equation (17) the discharge voltage should decrease as $m$ becomes larger.

The discharge voltage also depends strongly on the target material and the condition of the target (see section 5). The material dependence of the discharge voltage can be related to the value of the ion-induced electron emission yield $\gamma_{\mathrm{e}}$. Changing the target material and/or the target condition will result in a change in $\gamma_{e}$, and consequently the discharge voltage will change. As can be understood from equation (17), the discharge voltage is, to a first approximation, inversely proportional to the electron yield as shown in figure 12 .

\subsubsection{I-V characteristics}

One typical measurement when studying magnetron discharges, is the I-V characteristic. The behavior of the discharge voltage as a function of experimental parameters has been investigated in detail[32]. Another important parameter is the discharge current, as it is combined with the discharge voltage to provide power delivered to the target. At constant pressure, the discharge current increases rapidly with discharge voltage. To express this relationship, or the I-V characteristics, several empirical scaling laws have been proposed. The one used most often is

$\mathrm{I}=\mathrm{kV}^{\mathrm{n}}$.

The exponent $\mathrm{n}$ is a measure of the magnetron efficiency, and can have values of the order of 10 or higher. The behavior of the I-V characteristics as a function of the operating pressure is not fully understood. At low magnetic field strengths, the value of $n$ increases with increasing pressure, but the reverse behavior is apparent at high magnetic field strengths [32] as shown in figure 13.

The origin of this steep behavior is a current topic of discussion and research as it is related to the diffusion of electrons perpendicular to magnetic field lines. Based on the description given above, one could conclude that electrons can only escape from the magnetic trap by losing nearly all their energy in collisions. This is not completely true, as a result of frequent collisions with Ar atoms, electrons can be scattered and "hop" between field lines. This description is the classical diffusion of electrons based on collisions between electrons and gas atoms. However, experimental work shows that diffusion perpendicular to the magnetic field is greater than expected from the classical diffusion model[33]. Although several authors have proposed mechanisms to explain this enhanced diffusion, there are still doubts and unanswered questions.

\subsubsection{Magnet balance}

The magnet configuration in planar and rotating cylindrical magnetrons is used to trap electrons, thereby producing a high ion density near the target for a given pressure. With an optimum design of system geometry and operating pressure, the electrons are lost from the target area when their energy becomes less than the ionization threshold. Therefore, one can expect that only low-energy electrons are present in the bulk plasma region and in the vicinity of the substrate. This can be a distinct advantage to the glow discharge process, but in some cases (see further) it can also be advantageous to deliver more energy to the substrate. A possible solution is "unbalancing" the 
magnetron configuration, or changing the magnetic field strength of the inner versus outer magnets. In this way, the configuration of the magnetic trap is changed (see figure 13) to allow electrons to escape the magnetic trap follow the magnetic field lines towards the substrate. This results in a strong electron flux in the region of the substrate. By modifying the magnetic field strength balance between the outer and inner magnets, one can tune the electron flux, and hence the ion flux, incident at the substrate. One way to characterize the magnet balance is the ratio $\mathrm{K}$ between the magnetic flux through the outer and the inner magnets,

$\mathrm{K}=\frac{\Phi_{\text {out }}}{\Phi_{\text {in }}}=\frac{\int_{S_{\text {out }}} B_{\perp \text { out }} d S_{\text {out }}}{\int_{S_{\text {in }}} B_{\perp \text { in }} d S_{\text {in }}}$.

$\mathrm{B}_{\perp}$ represents the magnetic field strength perpendicular to the magnet, and $\mathrm{S}$ is the area of the magnet[34].

One way of changing the magnet balance is shifting the position of the inner and outer magnets relative to the target surface (see figure 15). This results in a different $n$ value for the I-V characteristic (see equation 18). Shifting the magnets also reduces the discharge voltage, which modifies the sputter rate of the target at constant current. The combination of both changes enables one to modify the ion-to-atom ratio at the substrate. The ion-to-atom ratio is a significant parameter during thin film growth (see section 7).

A more straightforward and completely tunable approach to unbalance the magnetic circuitry of the magnetron is to incorporate a Helmholtz coil external to the permanent magnets in order to increase the field strength of the outer magnet [35]. This opens a leak in the plasma trap and dramatically increases the incident ion/atom ratio at the substrate from typically 0.1-0.5 (depending on the gas, substrate bias, and system geometry) to $>50$ with no significant change in film deposition rates. Moreover, these high ion/atom ratios can be achieved using very low ion energies $(<10-20 \mathrm{eV})$ which, in most cases, are below the film damage threshold. Just as significantly, the ion energy and ion flux incident at the substrate are now controlled independently by the substrate bias and the external magnetic field, respectively. That is, the advantages of an ion beam deposition system are now obtainable using high rate magnetron sputtering.

\subsection{Powering the magnetron}

Magnetrons can be powered by a variety of methods. One can choose among radio frequency (rf), direct current (dc), pulsed dc, and, recently, high power impulse magnetron sputtering (HIPIMS)

\subsubsection{Radio frequency magnetron sputtering}

When using thick electrically non-conducting targets, $\mathrm{rf}$ power is the only option to sputter deposit thin films. An important point must be emphasized. The discussion of the magnetron sustaining mechanisms in sections 4.1 and 4.2 is not completely valid for rf powered magnetrons. Indeed, due to the rapidly changing electric field, the entire electron distribution shifts up in energy through "inphase" collisions, allowing either increased ionization (and, hence, discharge current) at constant pressure or operation at decreased pressure. The term "in-phase" collisions refers to the fact that there is a fraction of the electron population in the discharge that, while being accelerated by the instantaneous electric field, will make a collision before the field direction switches and will then have a velocity component in the direction of the switched field. Through this mechanism, the overall distribution of electrons increases in energy and, therefore, the concentration of electrons that have energies above the gas ionization potential increases, thus decreasing the overall discharge impedance.

When applying a high frequency (typically $13.56 \mathrm{MHz}$ ) power source between two electrodes, the light (low mass) electrons respond instantaneously to the rapidly varying field, while the heavier ions are inertially confined. That is, the ion transit time to either electrode is very much less than the rf period forcing both electrodes to charge negatively. In order to maintain charge neutrality per cycle, the entire rf waveform must then shift down in voltage such that each electrode is positive 
(attracting electrons) for less than 1\% of an rf period and the ions, to first order, respond to an average applied voltage which is approximately one-half the $\mathrm{rf}$ peak-to-peak potential. As in dc sputtering discussed above (section 4), the average ion impact energy is only a small fraction of the applied field due primarily to charge exchange collisions. In order to define the target and substrate in rf powered systems, one electrode (the substrate table) is made to be very much larger than the other electrode (the target) by electrically connecting the substrate table to the entire system. The electrodes now act as a leaky capacitively-coupled voltage divider for which the applied voltage is distributed as $V_{t} / V_{s} .=\left(A_{s} / A_{t}\right)^{m}$ where the subscripts $t$ and $s$ refer to target and substrate, $A$ is the electrode area and $\mathrm{m}=4$ in the ideal case, but is typically 1.5-2 for real systems.

An important lesson here is that when using $\mathrm{rf}$ power, one is always doing bias sputtering. It is intrinsic in the nature of how these systems function that there is an average negative voltage (and, hence ion bombardment) at the substrate. $\mathrm{V}_{\mathrm{s}}$ depends on the pressure, the gas, and system geometry (i.e., a smaller system confines the plasma and increases $V_{s}$ ). The substrate voltage can range from 15-20 V negative to a few hundred volts negative. It is also important to remember that since $\mathrm{rf}$ power supplies are designed to look into circuits with a purely resistive $50 \Omega$ load, a tunable matching network is required to damp out the complex reactance (both capacitance and inductance) of the discharge.

The primary disadvantage of the use of rf power supplies, in addition to expense and reliability, for magnetron sputtering is the inherently low deposition rate. That is, $\mathrm{n}$ decreases significantly in the power-law I-V relationship of equation 17. This is because magnetron sputtering, which relies on a closed electromagnetic trap at the target, is fundamentally a dc concept. The rf field alternately opens and closes the trap, allowing electrons to escape when the trap is open and forcing electrons to cross magnetic field lines, and hence drop power in the discharge, which decreases the available power at the target, when it is closed. The deposition rate, for a given applied target power decreases with both increasing frequency [36] and increasing magnetic field [37].

\subsubsection{Direct current magnetron sputtering}

The simplest and least expensive way to operate the magnetron is using a dc power supply. Today, special power supplies for sputter deposition have been developed which include an arc suppression unit. Arcs often occur during reactive sputter deposition of, for example, non-conductive oxides from a metal target in pure $\mathrm{O}_{2}$ or mixed $\mathrm{Ar} / \mathrm{O}_{2}$ discharges, due to a build up of oxide on the edges of the erosion groove where the sputter rate is low. Arcs (see figure 5) can be characterized as a low voltage and high current discharge. When the electric circuit "detects" a strong decrease of the discharge voltage and/or a strong increase of the discharge current, it switches the power off for a small period (typically a few microseconds) to draw electrons, decrease charging, and prevent the arc from fully developing. Arcing can seriously damage a target by local melting, but it also degrades quality of the deposited film due to the presence of particulates and/or pinholes while eventually destroying the power supply.

\subsubsection{Pulsed dc magnetron sputtering}

An effective way to prevent arcing during reactive magnetron sputtering (discussed below) is pulsing the applied voltage. In contrast to rf magnetron sputtering, the frequency is much lower and, is typically $50-250 \mathrm{kHz}$ depending upon the application[38]. Generally the discharge voltage cycles between a high negative voltage and a low positive voltage. The negative voltage part of the cycle is used to perform the sputtering, as the ions bombard the cathode. The low positive voltage part of the cycle is used to attract electrons to prevent charge buildup. Due to the higher mobility of the electrons, this part of the cycle can be relatively short compared to the total cycle time.

An alternative approach is the use of two magnetron sources, i.e. dual magnetron sputtering, and switch the negative and positive voltage between both targets. In this way, each magnetron alternately has the function of a sputtering target and an anode. Thus, both targets can be neutralized during each cycle. This not only prevents charge buildup but also solves problems related with anodes being covered with insulating thin films ("the disappearing anode"). The disadvantage here 
is the higher cost and the fact that power to each target must be turned off before igniting the other target when using mid frequency ac power supplies. Thus, transients are more severe in this approach. For pulsed dc dual magnetron sputtering, plasma ignition may be required after each halfcycle depending upon operation conditions. Dual magnetron power supplies also require electromagnetic and $\mathrm{rf}$ interference shielding.

\subsubsection{High power impulse magnetron sputtering}

HIPIMS uses a large energy impulse supplied to the cathode over a very short time period, typically $\sim 100 \mu \mathrm{s}$. This requires a very different type of power supply, the heart of which is a large bank of capacitors. The HIPIMS process delivers a large low energy flux of ions to the substrate. Peak powers up to several $\mathrm{MW} /$ pulse with pulse widths between 100 and $150 \mu$ s must be generated by the power supply. Average powers are $\sim 20 \mathrm{~kW}$ with frequencies up to $500 \mathrm{~Hz}$. In addition to supplying pulsed power, arc suppression is also necessary. This process takes advantage of enhanced ionization resulting from the high energy pulse. Power densities applied to the target are in the neighborhood of $1-3 \mathrm{~kW} / \mathrm{cm}^{2}$ compared to traditional magnetron sputtering with power densities $\sim 1-10 \mathrm{~W} / \mathrm{cm}^{2}$.

The high power pulse is the core of this process. Typical operating conditions are as follows. The voltage pulse is between $1.3-1.5 \mathrm{kV}$ and total pulse duration is $\sim 120 \mu \mathrm{s}$. The target current increases and peaks at $\sim 200 \mathrm{~A}$ as the voltage pulse decays. The power density at that point is $\sim$ $600 \mathrm{~W} / \mathrm{cm}^{2}$. This method of powering the target results in a high density plasma with a high ionization degree of the sputtered target material. During each sputtering pulse, the discharge begins as gas-supported, but due to the huge powers quickly becomes metal-supported (self-sputtering) due to gas rarefaction. HIPIMS has recently been reviewed in detail in [39].

\section{Reactive magnetron sputter deposition}

Using an electrically conducting, and generally, a metallic target makes powering the magnetron much easier as several alternatives are now possible. However, it limits the choice in the materials that can be deposited with this technique. To mitigate this problem, a reactive gas can be added to the discharge. The reactive gas, e.g. nitrogen, oxygen, hydrogen sulphide, etc. can react with the sputtered material to form a compound on the substrate. However, the addition of the reactive gas to the discharge influences the deposition process in several ways while increasing the complexity of the overall process. Some aspects of the reactive sputter deposition processes are discussed in this section.

\subsection{Hysteresis of reactive gas pressure and discharge voltage}

To add or remove a reactive gas from the discharge, one generally uses a flow controller. A typical experiment is to stepwise increase the reactive gas flow over a given interval, and wait between the steps until the process is stable, at which time the process parameters (pressure, voltage, current, deposition rate) are recorded. After reaching a maximum flow, the reactive gas flow is stepwise decreased, and again one gives the system sufficient time to stabilize after each step. At sufficiently low pumping speed and high discharge current one notices that at a given reactive gas flow, for many reactive gas/target material combinations, there is an abrupt change in the total pressure and discharge voltage as shown in figure 16. One also notices that the reactive gas flow must be decreased to a much lower value than the critical value detected during reactive gas addition. Or stated differently, there is a hysteresis in both reactive gas pressure and discharge voltage. The low oxygen flow region is defined as "metal mode", and is characterized by a low reactive gas partial pressure and a high deposition rate. The deposited layer is not a pure compound, but rather a doped metal. The high oxygen flow region is defined as the "compound mode", or "poisoned mode", with a high partial pressure and a low deposition rate. The film is essentially a pure compound. 


\subsection{Understanding the hysteresis behavior: modeling the reactive sputter process}

To understand the hysteresis behavior, several authors have modeled the process. The most well known, the"Berg" model, is named after S. Berg from Uppsala University[40]. The model has value due to its simplicity and the fact that it captures much of the physics of the process. As stated by Berg et al., the results should be considered as a first order approximation. Nevertheless, some authors state that the model fits their experimental results. This statement is dangerous, because the fitted parameters are often not experimentally verified, or have no physical meaning. Some experimental results can be better explained by changing the description of the processes occurring at the target, i.e. by including reactive ion implantation. This was first proposed by Depla et al. [41][42], and the combined model will be briefly reviewed in this chapter. A user-friendly, shareware code, entitled RSD2008[43], is available.

The model is based on balance equations, an approach followed by many authors, and originally proposed by Berg et al. The first balance equation is related to the flow,

$\mathrm{q}_{0}=\mathrm{q}_{\mathrm{P}}+\mathrm{q}_{\mathrm{T}}+\mathrm{q}_{\mathrm{S}}$

and states that the reactive gas flow introduced into the vacuum system $\left(q_{o}\right)$ must equal the sum of the reactive gas flows to the pump $\mathrm{q}_{\mathrm{p}}$, and the number of reactive gas molecules consumed per unit time in reactions at the target $\left(\mathrm{q}_{\mathrm{T}}\right)$, and at the substrate $\left(\mathrm{q}_{\mathrm{S}}\right)$. The reactive gas flow toward the pump depends solely on the partial pressure of the reactive gas $\mathrm{P}$ and the pumping speed $\mathrm{S}$, and can be expressed as

$\mathrm{q}_{\mathrm{P}}=\mathrm{PS}$.

The reaction on the substrate between the reactive gas molecules and the deposited target material is described as by Berg et al., as a chemisorption process. Hence, the flow towards the substrate will depend on the reactive gas coverage at the substrate, $\theta_{\mathrm{s}}$. It will also depend on the sticking coefficient of the reactive gas $\alpha_{s}$ and the substrate area $A_{s}$. The latter expresses the area of all surfaces on which deposition occurs. Hence, the term "substrate" refers to the entire system except the target. Summarizing,

$\mathrm{q}_{\mathrm{S}}=\alpha_{\mathrm{s}} \mathrm{F}\left(1-\theta_{\mathrm{s}}\right) \mathrm{A}_{\mathrm{s}}$

with $\mathrm{F}$ the flux of reactive gas molecules towards the substrate. This latter term is defined as

$$
\mathrm{F}=\frac{\mathrm{P}}{\sqrt{2 \pi \mathrm{mkT}}}
$$

with $\mathrm{m}$ the mass of the reactive gas molecule, $\mathrm{k}$ the Boltzmann constant, and $\mathrm{T}$ the gas temperature. The reaction at the target cannot be described solely by reactive gas chemisorption. One must include other reaction paths. In the model proposed by Depla et al., two mechanisms are included, i.e. direct reactive ion implantation and knock-on of chemisorbed atoms into the target. Including these extra reaction paths makes the model more complex but strongly enhances the understanding of reactive magnetron sputtering. In the model, one distinguishes between the surface layer and the subsurface region. Using this description of the target, the steady-state flow towards the target can be written as,

$$
\mathrm{q}_{\mathrm{t}}=\left(\mathrm{F} \alpha \theta_{\mathrm{sm}}-\frac{\mathrm{I} \beta \theta_{\mathrm{sc}}}{2}+\frac{\mathrm{z}}{2} \mathrm{Y}_{\mathrm{s}} \theta_{\mathrm{rb}}\right) \mathrm{A}_{\mathrm{t}}
$$

with $A_{t}$ the target surface area and $z$ defined by the stoichiometry of the compound $M_{\mathrm{z}}$ with $\mathrm{M}$ the metal and $\mathrm{R}$ the reactive gas. Equation (24) takes into account chemisorption of the reactive gas on the metal fraction of the target, $\theta_{\mathrm{sm}}$. Knock-on of chemisorbed atoms into the target will depend on the ion current density I, the knock-on yield $\beta$, and the fraction of the target covered with chemisorbed oxygen atoms $\theta_{\mathrm{sc}}$. Finally, direct reactive ion implantation is also included. In this mechanism, the compound is formed in the subsurface region of the target and, due to target 
erosion, it reaches the surface. The fraction of the compound in the subsurface region, i.e. just beneath the surface layer, is expressed as $\theta_{\mathrm{rb}}$. The target erosion is defined by the ion current density and the total sputter yield of the target $Y_{s}$, which of course depends on the surface composition,

$\mathrm{Y}_{\mathrm{s}}=\theta_{\mathrm{sm}} \mathrm{Y}_{\mathrm{m}}+\theta_{\mathrm{sc}} \mathrm{Y}_{\mathrm{c}}+\theta_{\mathrm{sr}} \mathrm{Y}_{\mathrm{r}}$

with $\theta_{\mathrm{sr}}$ the compound surface fraction and $\mathrm{Y}_{\mathrm{r}}$, the compound yield. Clearly,

$\theta_{\mathrm{sm}}+\theta_{\mathrm{sc}}+\theta_{\mathrm{sr}}=1$.

The next step in the model is to calculate the different surface and bulk fractions of compound and unreacted target material. We need also to calculate the reactive gas substrate coverage, $\theta_{\mathrm{s}}$. This latter term is perhaps the easiest to start with. Essentially three processes occur at the substrate:

- Chemisorption of reactive gas on the metal fraction of the substrate

- Sputter deposition of compound onto the substrate

- Sputter deposition of metal onto the substrate.

These three processes lead to the following steady state equation,

$$
\frac{2 \mathrm{~F}}{\mathrm{z}} \alpha_{\mathrm{c}}\left(1-\theta_{\mathrm{s}}\right)+\frac{\left(\mathrm{Y}_{\mathrm{c}} \theta_{\mathrm{sc}}+\mathrm{Y}_{\mathrm{r}} \theta_{\mathrm{sr}}\right) \mathrm{IA} \mathrm{A}_{\mathrm{t}}}{\mathrm{A}_{\mathrm{c}}}\left(1-\theta_{\mathrm{s}}\right)-\frac{\mathrm{Y}_{\mathrm{sm}} \theta_{\mathrm{sm}} \mathrm{IA} \mathrm{A}_{\mathrm{t}}}{\mathrm{A}_{\mathrm{c}}} \theta_{\mathrm{s}}=0
$$

For simplicity, compound sputtering is described as molecular, but in reality the compound will leave the target primarily as atoms.

Implicitly, this equation assumes a uniform deposition profile, which is of course too simple. By subdividing the substrate in different areal cells, with areas $A_{c, i}$ and calculating the metal and compound flux arriving at each substrate cell using a transport code (see next section), one can describe the condition of the substrate locally by solving equation (21) for $\theta_{\mathrm{s}, \mathrm{i}}$, the condition of each substrate cell. This gives a much better description of the substrate composition as a function of the oxygen flow.

Similarly, we describe the surface and bulk condition of the target. At the surface, three processes are described by the model: chemisorption, knock-on, and sputtering, which leads to the following relations,

$$
\begin{aligned}
\frac{2 \mathrm{~F}}{\mathrm{z}} \alpha \theta_{\mathrm{ms}} & =\mathrm{Y}_{\mathrm{c}} \theta_{\mathrm{cs}} \mathrm{I}+\frac{\mathrm{I}}{\mathrm{z}} \beta \theta_{\mathrm{cs}} \\
\frac{\mathrm{I}}{\mathrm{z}} \beta \theta_{\mathrm{cs}}+\mathrm{IY} \theta_{\mathrm{s}} \theta_{\mathrm{mb}} & =\mathrm{Y}_{\mathrm{m}} \theta_{\mathrm{ms}} \mathrm{I}+\frac{2 \mathrm{~F}}{\mathrm{z}} \alpha \theta_{\mathrm{ms}} \\
\mathrm{v}_{\mathrm{s}}(\mathrm{t}) \theta_{\mathrm{rb}} \mathrm{n}_{\mathrm{o}} & =\mathrm{I} \theta_{\mathrm{rs}} \mathrm{Y}_{\mathrm{r}} .
\end{aligned}
$$

The first equation describes the balance between chemisorption (left side) and sputtering of the chemisorbed atoms and knock-on events (right side). The second equation describes the metal surface balance. Knock-on processes of chemisorbed atoms result in an increase in the metal surface fraction. We also must take into account that metal from the bulk is transported toward the surface due to target erosion. At the right side, two mechanisms leading to a decrease of the metal fraction are described, i.e. sputter removal and chemisorption. The third equation describes the balance between sputter removal of compound material and the transport of compound from the subsurface region to the surface region, defined by the target erosion speed $\mathrm{v}_{\mathrm{s}}$.

To include the reaction of the implanted and knock-on implanted reactive gas atoms with the target material, one needs to account for target erosion, implantation, and chemical reaction. This leads to the following equations,

$$
\begin{aligned}
& \frac{\partial \mathrm{n}_{\mathrm{r}}(\mathrm{x}, \mathrm{t})}{\partial \mathrm{t}}=2 \mathrm{fIp}(\mathrm{x})-\mathrm{kzn}_{\mathrm{r}}(\mathrm{x}, \mathrm{t}) \mathrm{n}_{\mathrm{m}}(\mathrm{x}, \mathrm{t})+\mathrm{I} \theta_{\mathrm{cs}} \beta \mathrm{p}_{\mathrm{c}}(\mathrm{x})-\mathrm{v}_{\mathrm{s}}(\mathrm{t}) \frac{\partial \mathrm{n}_{\mathrm{r}}(\mathrm{x}, \mathrm{t})}{\partial \mathrm{x}} \\
& \frac{\partial \mathrm{n}_{\mathrm{m}}(\mathrm{x}, \mathrm{t})}{\partial \mathrm{t}}=-\mathrm{kn}_{\mathrm{r}}(\mathrm{x}, \mathrm{t}) \mathrm{n}_{\mathrm{m}}(\mathrm{x}, \mathrm{t})-\mathrm{v}_{\mathrm{s}}(\mathrm{t}) \frac{\partial \mathrm{n}_{\mathrm{m}}(\mathrm{x}, \mathrm{t})}{\partial \mathrm{x}} .
\end{aligned}
$$


The first describes the time evolution of the concentration $n_{r}$ of non-reacted implanted reactive gas atoms, while the second does the same for the concentration $\mathrm{n}_{\mathrm{m}}$ of non-reacted target atoms. The amount of reactive gas atoms which becomes implanted depends on the mole fraction $f$ of reactive gas molecules in the discharge. The directly implanted reactive atoms are distributed over the subsurface region according to the implantation profile $\mathrm{p}(\mathrm{x})$. A similar approach can be used to describe the distribution of knock-on implanted atoms, i.e. $\mathrm{p}_{\mathrm{c}}(\mathrm{x})$. Due to target erosion, there is material transport toward the target surface (last term in both equations). The chemical reaction between both the reactive gas atoms and the target atoms is defined by their concentrations and the reaction rate constant $\mathrm{k}$. In steady state, these equations simplify to

$$
\begin{aligned}
& \mathrm{v}_{\mathrm{s}} \frac{\partial \mathrm{n}_{\mathrm{r}}(\mathrm{x}, \mathrm{t})}{\partial \mathrm{x}}=2 \mathrm{fIp}(\mathrm{x})-\mathrm{kzn}_{\mathrm{r}}(\mathrm{x}) \mathrm{n}_{\mathrm{m}}(\mathrm{x})+\mathrm{I} \theta_{\mathrm{cs}} \beta \mathrm{p}_{\mathrm{c}}(\mathrm{x}) \\
& \mathrm{v}_{\mathrm{s}} \frac{\partial \mathrm{n}_{\mathrm{m}}(\mathrm{x}, \mathrm{t})}{\partial \mathrm{x}}=-\mathrm{kn}_{\mathrm{r}}(\mathrm{x}) \mathrm{n}_{\mathrm{m}}(\mathrm{x}) .
\end{aligned}
$$

The cascade of equations in the model can be solved quite easily by iteration. One assumes a given target subsurface reaction, i.e. $\theta_{\mathrm{rb}}$. For a given partial pressure of reactive gas, one can calculate, using equations (28), the target surface condition and the erosion rate. This enables the solution of equations (30), which describe the target subsurface condition. At steady state, the calculated reaction state should equal the initial value. If the value obtained is too high, the partial pressure (or the mole fraction), is altered, and the calculation is repeated. With the calculated surface and subsurface conditions, it is possible then to calculate the flow towards target, substrate, and pump. By summing these three flows, the target condition, the substrate condition, and the partial pressure as a function of the reactive gas flow can be calculated. Or stated differently, a hysteresis experiment can be simulated. To facilitate the use of this model, a downloadable program was written, which predicts the influence of different parameters on the hysteresis curves[43]. Essentially the program provides an output as shown in figure 17. Comparing simulation with experimental results (see figure 14) enables the understanding of the origin of the hysteresis effect. At low reactive gas flow, the partial pressure of the reactive gas, oxygen in this example, is negligible because most of it is consumed by the metal deposited on the substrate. Indeed, as figure 17 shows, the target composition remains almost unaffected (middle panel), especially in the subsurface region. Preferential sputtering enriches the surface region with the compound and, together with the chemisorption of oxygen, results in substantial surface oxidation. The difference between unaffected subsurface and oxidized surface regions can explain the small initial increase in discharge voltage noticed in the experiment during oxygen addition in the metal mode (see figure 16). The oxidation of the surface region results in a decrease in the total sputter yield, as the compound material sputters less efficiently than the metal due to a higher surface binding energy. Hence, less metal is deposited, and more reactive gas becomes available for target oxidation. An avalanche situation develops which results in complete and rapid oxidation of the substrate and the target. This also causes an abrupt change in the partial pressure and other deposition parameters such as discharge voltage. The simulation shows that within the hysteresis region (dark shaded area), the slope of the partial pressure vs. flow curve is negative. Hence, this region is not stable under flow control. To return to metal mode from the compound mode, one needs to lower the oxygen flow to a value smaller than the critical flow measured during the flow increase. This behavior is due to the large difference in sputter yield between the metal and the compound.

For rotating cylindrical magnetron, the hysteresis is also a function of the rotation speed. Figure 18 shows the behavior of the critical point as function of the rotation speed for the laboratory scale rotating magnetron depicted in figure 11.

Understanding this behavior requires a time dependent, rather than a steady state, description of the reactive sputter process, because, due to target rotation, processes which occur outside the race track influence the process as that region rotates into the discharge. Moreover, an additional process must be taken into account, i.e. redeposition of sputtered material onto the cathode [44]. 


\subsection{Circumventing the hysteresis problem.}

Hysteresis behavior hinders the application of reactive magnetron sputter deposition. Indeed, when controlling the process by regulating the flow, one notices that the smallest change in the reactive gas flow and/or other deposition conditions, can result in a run-a-way situation from metal mode to compound mode. Optimal use of reactive magnetron sputter deposition appears to require using a reactive gas flow close to the edge of the metal mode region, as this combines nearly stoichiometric compound layers with a high deposition rate. The problem with this is that minor perturbations in the discharge, which always occur, are amplified and move the system beyond its metastable operation point. However, several solutions are available. A first solution, as described by Berg et al.[40], requires a dramatic increase in pumping speed. In this situation, hysteresis will be avoided, as the reactive gas partial pressure in the vacuum chamber is to a large extent controlled by the pump, and not by the deposition process. However, this is not a cost effective solution. An alternative approach was proposed by Sproul et al.[38]. The reactive gas flow is controlled by a feedback system which measures the partial pressure of the reactive gas, the optical emission spectrum of the metal, or the discharge voltage. So, one switches from flow control to partial pressure control. Indeed, as simulation results show (see figure 17), if one interchanges pressure and flow axes, full control is possible and one can combine complete compound formation with a relatively high deposition rate. The disadvantage of this approach is the complexity and the cost of the equipment. Another solution is using conductive substoichiometric targets (e.g. $\mathrm{TiO}_{2-\mathrm{x}}$ with x of the order of 0.2 [45]) for which only a low reactive gas flow is required, and generally the deposition process does not show a hysteresis behavior. However, substoichiometric target processing is more expensive and complicated as these targets are mostly ceramics. In conclusion, circumventing the hysteresis problem is possible, but it always comes with an additional cost and/or increased system complexity.

\section{Moving towards the substrate}

\subsection{Sputtered particles}

All sputtered particles leaving the target can collide with the gas atoms present in the vacuum chamber during transit to the substrate. The same is true for ions which are neutralized and reflected at the target, and for negative ions formed at the target [46]. Collisions will alter particle energy, direction, and momentum, and therefore also the morphology and microstructure of the growing film (see next section). The most important parameter influencing gas phase transport is pressure.

Several models have been proposed[47] to simulate the transport of sputtered particles. Recently, K. Van Aeken et al.[48] have developed a user-friendly shareware model SiMTRA (SiMulation of TRAnsport). This flexible model enables calculation of the energy, direction, and flux of sputtered particles incident on every defined surface in the vacuum chamber. The output is not only the deposition profile, but also the energy of the arriving particles on the substrate. The output of the code can be combined with the reactive sputter deposition model RSD2008, described in the previous chapter, enabling a more detailed description of the reactive sputtering process. Figure 19 shows the result of such a calculation. Oxidation of the substrate, here a tube with the magnetron source located at the top center of the tube, is tracked as a function of the oxygen flow. At low oxygen flows, only the material deposited at the bottom of the tube is fully oxidized (red). As the oxygen flow increases, all deposited material becomes oxidized. The transition from metal mode to compound mode occurs when the deposited material is nearly fully oxidized.

The SiMTRA model provides insight into the mechanisms controlling the transport of sputtered particles towards the substrate (see figure 20). The ejection positions of the sputtered particles are based on the (simulated) race track of the magnetron. One can use the Thompson equation (see equation (5)) to describe the energy distribution of the sputtered particles, and the nascent angular distribution can be calculated using SRIM or can be based on experimental input. The model assumes that the sputtered particles are neutral atoms in the ground state, and these atoms only undergo elastic collisions with neutral gas atoms. For dc magnetron sputtering, this is a reasonable 
approach for two reasons. First, the ionization degree is generally very low. Most authors report an ionization degree of the order $0.1 \%$ and lower (see e.g. [33][49][50]). Secondly, the sputtered particle density is much less than the gas density. Of course, the ratio between the sputtered particle density, and the gas density depends on the pressure and the power but is of the order of $10^{-4}$ (see e.g. [50][51]). Based on these assumptions, the path of the sputtered atom can be described as a sequence of straight trajectories each terminated by a binary elastic collision with a gas atom, until deposition on a surface occurs. Classical scattering theory is followed [27]. The free path length $\lambda$ until the next collision is derived from a mean free path length $\lambda_{\mathrm{m}}$ as

$\lambda=-\lambda_{\mathrm{m}} \ln r_{1}$

with $r_{1}$ a random number uniformly chosen over the range $[0,1]$. The description of the mean free path length depends on the velocity of the sputtered particles $\mathrm{v}_{\mathrm{s}}$ compared to the velocity of the gas atoms $\mathrm{v}_{\mathrm{p}}$. At high velocity $\left(\mathrm{v}_{\mathrm{s}}>5 \mathrm{v}_{\mathrm{p}}\right)$, the gas atoms are considered stationary. When $\mathrm{v}_{\mathrm{s}}$ drops below $5 \mathrm{v}_{\mathrm{p}}$ the thermal motion of the gas atoms is taken into account. Finally when the energy of the sputtered particles falls below $3 / 2 \mathrm{kT}$ the sputtered particle is considered thermalized.

\subsection{Other particles arriving at the substrate}

Besides the arrival of sputtered particles (see section 6.1), the flux of other particles arriving at the substrate is also important as these particles determine the total energy and momentum flux towards the substrate and hence they play a crucial role in the thin film growth (see section 7). The total energy flux per incoming metallic particle $\mathrm{E}_{\text {tot }}$ can be described as a linear summation of different contributions [52-56].

$\mathrm{E}_{\text {tot }}=\mathrm{E}_{\text {cond }}+\mathrm{E}_{\mathrm{pl}}+\mathrm{E}_{\mathrm{t}}+\mathrm{E}_{\mathrm{sp}}+\mathrm{E}_{\text {refl }}+\mathrm{E}_{\text {gas }}+\mathrm{E}_{\mathrm{el}}+\mathrm{E}_{\text {ion }}$,

as summarized in Table 2.

\begin{tabular}{|c|c|c|}
\hline Particle & $\begin{array}{l}\text { Energy } \\
\text { contribution }\end{array}$ & Description and comments \\
\hline \multirow[t]{2}{*}{ Photons } & $\mathrm{E}_{\mathrm{pl}}$ & $\begin{array}{l}\text { The energy flux due to plasma radiation. As discussed in section ( } 4.1) \text {, the average } \\
\text { energy per ionisation } \mathrm{W} \text { is approximately } 30 \mathrm{eV} \text { in an Ar discharge. However, the actual } \\
\text { ionization energy of } \mathrm{Ar} \text { is only } 15.76 \mathrm{eV} \text {. Hence, approximately } 14 \mathrm{eV} \text { will be transferred } \\
\text { to the plasma per ionized Ar. }\end{array}$ \\
\hline & $\mathrm{E}_{\mathrm{t}}$ & $\begin{array}{l}\text { The energy flux due to thermal radiation from hot bodies in the vacuum deposition } \\
\text { system. }\end{array}$ \\
\hline \multirow[t]{4}{*}{$\begin{array}{l}\text { Atoms } \\
\text { Molecules }\end{array}$} & $\mathrm{E}_{\mathrm{sp}}$ & $\begin{array}{l}\text { The energy flux due to the kinetic energy of the sputtered particles which, as shown in } \\
\text { section } 2 \text {, is substantially larger then the thermal energy (see figure } 4 \text { ). }\end{array}$ \\
\hline & $\mathrm{E}_{\text {cond }}$ & $\begin{array}{l}\text { The energy flux due the formation of a compound by a chemical reaction on the substrate } \\
\text { and/or the condensation of a metallic species on the substrate }\end{array}$ \\
\hline & $\mathrm{E}_{\mathrm{refl}}$ & $\begin{array}{l}\text { The energy flux due to neutralized and reflected working gas atoms. Just prior to impact } \\
\text { at the target, a high energy ion will be neutralized and it can be reflected towards the } \\
\text { substrate. }\end{array}$ \\
\hline & $\mathrm{E}_{\text {gas }}$ & $\begin{array}{l}\text { The energy flux due to working gas atoms. Normally this contribution can be neglected, } \\
\text { but the interaction of the sputtered particles with the gas (see section } 6.1 \text { ) can result in } \\
\text { gas heating, also known as gas rarefaction [57][58]. }\end{array}$ \\
\hline Electrons & $\mathrm{E}_{\mathrm{el}}$ & Energy flux due to incident electrons \\
\hline Ions & $\mathrm{E}_{\mathrm{ion}}$ & Energy flux due to the ion flux towards the substrate \\
\hline
\end{tabular}

Table 2. Different contributions to the energy flux toward the substrate

Based on a combination of modeling, and measurements with Langmuir probe, retarding field analyzer, quartz microbalance, and energy resolved mass spectrometer, Mahieu et al. have quantified the total energy flux and show the relationship with the crystallographic orientation and microstructure of deposited TiN films [59][60]. This connects the energy (and momentum) flux to 
properties of the films (such as hardness), and to deposition conditions. In the next section, we focus on the relationship between deposition conditions and microstructure.

\section{Sputter deposited thin films: morphology and microstructure}

Several authors have attempted to summarize the influence of deposition parameters on film morphology and microstructure in a single diagram. These diagrams are known as "structure zone models" (SZMs). The SZM best known in the sputter deposition community is the one published by Thornton [61], which shows microstructure and morphology as a function of deposition pressure and substrate temperature. This historically important model forms the basis of several studies, see for example[62]. Recently, Mahieu et al. [63] published an extended SZM (ESZM) which has the advantage that it not only summarizes the influence of deposition parameters on film microstructure, but also provides insight into sputter deposited thin film growth. Hence, film structure (microstructure, and crystallographic orientation) is plotted as a function of adatom mobility which depends on the energy flux per sputtered particle (see previous section); which in turn depends on the deposition parameters. To quantify the ESZM, it is necessary to calculate all energy flux contributions towards the substrate. In this section, we will discussed the ESZM.

\subsection{Zone I films}

At low energy per incident particle, adatom mobility is low, and each particle will stick to the growing film at more or less the position at which it arrives. Stated differently, the adatoms have a low probability to overcome the existing diffusion barriers. Hence, one refers to this part of the ESZM, as the "hit and stick" region. Formation of large compact, crystalline islands is therefore not possible and only small crystallites can develop. Film structure has an amorphous-like appearance, and its crystallographic orientation is not preferential. The films are rough due to statistical, or kinetic, roughening and self-shadowing. Even for normal adatom incidence, overhang structures can be formed, shadowing the underlying layers and preventing further local growth. Such films typically have reduced density, high porosity and are classified as Zone Ia films.

Film density can be improved by bombardment of energetic species (ions, fast neutrals, sputtered particles, and neutralized reflected ions). Bombardment results in knock-on events that destroy the overhang structures of Zone Ia films. Voids between the columns of a Zone Ia film begin to be filled, and a more dense columnar structure evolves. As the energetic bombardment only results in "reorganisation", the structure still has an amorphous-like appearance. This type of film can also be grown by using an ion beam incident at the substrate during thermal evaporation. The change in microstructure from Zone Ia to Zone Ib is not possible by just increasing the substrate temperature alone.

When the energy flux toward the substrate is increased, the adatoms will become more mobile, resulting in compact crystalline islands. So, in contrast to Zone Ia and Ib, the grains in Zone Ic will be facetted. Crystallographic diffraction scans show that only the crystal planes with lowest crystallographic normal growth rate will survive, and therefore the grains in Zone Ic are terminated by these planes. The normal growth rate is strongly influenced by many parameters, but under common deposition conditions and low energetic bombardment, two parameters are important: the sticking coefficient of the adatoms vs. crystallographic orientation and adatom mobility. A higher sticking coefficient results in a higher normal growth rate. The influence of the mobility is more complex. If the mobility is high, the adatom can reach the edge of the crystal or grain, where it can descend the step (depending on the Ehrlich barrier height). Hence, a high mobility is conducive to lateral growth, while low mobility results in a higher normal growth rate of the crystal plane. In the case of a rather low energy flux, what is characteristic for zone Ic, the probability that adatoms diffuse from one grain to another is low, due to the small diffusion length. Hence, neighboring grains will not interchange adatoms and thus will not interact with each other. This results in a microstructure consisting of straight, clearly faceted columns as each grain grows independently in a direction perpendicular to the substrate. There is no competition among the growing columns. However, the tallest columns will capture more sputtered atoms and can shadow the adjacent 
columns. Nevertheless, this overgrowth mechanism is slow and, as a result, little preferential out-ofplane orientation is observed.

\subsection{Zone T films}

Further increasing the adatom mobility results in a microstructure arising from adatoms diffusing between adjacent grains This results, in turn, in an overgrowth mechanism, different from the shadowing mechanism discussed in the previous section. Initially, well-faceted grains will form on the substrate. The facets are defined by the planes with lowest normal growth rate. The out-of-plane orientation of these grains will be different, if nucleation occurs randomly. Let us focus on two differently oriented grains. In zone $\mathrm{T}$, the arriving adatoms have a high surface mobility, and can therefore diffuse from one grain to the other. Upon impingement of the two grains, a grain boundary is created. The position at the substrate of the grain boundary is fixed because restructuring grain growth (see zone II for a definition) is not possible under zone T conditions.

However, the direction of this grain boundary can change during the film growth due to evolutionary growth. As depicted in figure 21, the grains with the most tilted facets with respect to the substrate plane will overgrow other grains because they have the fastest geometric growth rate in the direction perpendicular to the substrate. This has an important consequence. At a given layer thickness, all grains merge toward the same crystallographic orientation. Thus, zone T films have properties which vary with thickness. So, summarizing this mechanism: due to an evolutionary overgrowth, a film structure is obtained will a clearly facetted surface, V-shaped columns, and a preferential crystallographic orientation., corresponding to the orientation with the fastest geometric growth rate.

\subsection{Zone II films}

The specific microstructure of zone $\mathrm{T}$ films is kinetically determined. At higher energy flux, additional processes can occur which one can summarize as restructuring grain growth. This includes processes such as ripening, cluster diffusion or grain boundary migration. The final result of these processes is actually the same, i.e. from the nucleation stage on, film growth evolves along a path which minimizes the total system energy. These restructuring grain growth processes are not only driven by the minimization of the island or grain surface area, but also by the minimization of the surface and interface energy and anisotropy in elastic constants (hence in strain energy density versus orientation) The first result is that the film consists of straight columns with a curved surface. As a result, column tops will, depending on the column diameter, not be perfectly flat but slightly curved. A second important consequence of restructuring grain growth is the development of a preferential out-of-plane crystal orientation. Surface energy depends on the specific crystallographic plane. Hence, if restructuring grain growth drives the film to minimize the total surface energy, grains with a high energy plane parallel to the substrate will be consumed by grains with a low energy plane parallel to the substrate. Therefore, the resulting preferred out-of-plane orientation is the crystallographic orientation perpendicular to the plane of lowest surface energy. Due to high surface diffusion and restructuring grain growth, shadowing will have a minor influence on growth. Hence, tilting the substrate to the incoming material flux will not cause significant inclination of the columns. Further increasing the energy flux increases the effects of restructuring grain growth. Thus, the columns forming the film will increase their diameter.

\section{Conclusions}

Several aspects of the sputter deposition process are still not completely understood yet. Fundamental points related to the sputter process and the discharge include: the quantification of the sputter yield of compounds, the emission of negative species from oxide and oxidized targets, the description of high energy electron diffusion perpendicular to the magnetic field, the understanding of the discharge voltage behaviour during reactive magnetron sputtering. New trends, such as HIPIMS, bring new questions and problems. Hence, there is an on-going research towards a better description of this technique. However, many of the fundamental issues which are not yet 
understood do not hinder the application of this interesting technique. This explains the wide application field for sputter deposition.

\section{Reference}

[1] The Web of Science is part of the ISI Web of Knowledge Platform produced by Thompson Scientific. It can be accessed via http://apps.isiknowledge.com/.

[2] The patent database Delphion is part of the Thomson Corporation. It can be accessed via http://www.delphion.com.

[3] G. Carter and J.S. Colligan, Ion Bombardment of Solids, American Elsevier, New York (1968).

[4] L.I. Maissel, "Applications of Sputtering to the Deposition of Films," in Handbook of Thin Film Technology, ed. by L.I. Maissel and R. Glang, McGraw-Hill, New York (1970).

[5] G.K. Wehner and G.S. Anderson "The Nature of Physical Sputtering," in Handbook of Thin Film Technology, ed by L.I. Maissel and R. Glang, McGraw-Hill, New York (1970).

[6] M. Nastasi, J. Mayer, J.K. Hirvonen, "Ion-Solid Interactions: Fundamentals and Applications," Cambridge Solid State Science Series, Cambridge University Press, Cambridge, UK (2004), (ISBN: 9780521616065).

[7] "Sputtering by Particle Bombardment: Experiments and Computer Calculations from Threshold to MeV Energies," Edited by R. Behrish and W. Eckstein, Springer, Berlin, Germany (2007). (ISBN: 9783540445005).

[8] R.A. Baragiola, Phil. Trans. R. Soc. Lond. A (2004) 362, $29-53$.

[9] V. S. Smentkowski, Progress in Surface Science 64 (2000) 1-58.

[10] W.D. Westwood „Sputter deposition“ AVS Education Committee Book Series, Vol. 2, AVS New York ISBN 10-0-735-0105-5.

[11] H.H. Andersen, H.L. Bay, Sputter yield measurments in Topics in Applied Physics, volume 47, Sputtering by Particle Bombardment I, Editor R. Behrish, Springer-Verlag Berlin Heidelberg New York.

[12] Sigmund, Phys. Rev. 184 (1969) $383-416$.

[13] "Simulation of the sputtering process", T. Ono, T. Kenmotsu, T. Muramoto in "Reactive Sputter deposition”, D. Depla, S. Mahieu (editors), Springer Berlin Heidelberg New York 2008, ISBN 978-3-54076662-9.

[14] Y. Yamamura, H. Tawara, At. Data Nucl. Data Tables 62 (1996) 149.

[15] J.E. Mahan, A. Vantomme, J. Vac. Sci. Technol. A 15 (1997) 1976-1989.

[16] Stopping and Range of Ions in Matter can be downloaded from www.srim.org.

[17] Stopping and Range of Ions in Matter, J.F. Ziegler, J.P. Biersack, M.D. Ziegler, ISBN 978-0-9654207-1-6.

[18] W. Möller, W. Eckstein, Nucl. Instr. Methods in Phys. Res. B 2 (1984) 814-818.

[19] M.W. Thompson, Vacuum 66 (2002) 99-114.

[20] G. Falcone, Phys. Rev. B 28 (1988) 6398-6401.

[21] J.B. Malherbe, S. Hoffmann, J.M. Sanz, Appl. Surf. Sci. 27 (1986) 355-365.

[22] A. Anders Surface \& Coatings Technology 200 (2005) $1893-1906$.

[23] W.R. Grove, Phil. Trans. R. Soc. Lond. 142 (1852) 87-101.

[24] W.D. Davis, T.A. Vanderslice, Phys. Rev. 131 (1963) 219.

[25] M. Van Straaten, A. Bogaerts, R. Gijbels, Spectrochimica Acta B 50 (1995) 583-605.

[26] A. Bogaerts, M. Van Straaten, R. Gijbels, Spectrochimica Acta B 50 (1995) 179-196.

[27] M.A. Lieberman, A.J. Lichtenberg, Principles of plasma discharges and Materials Processing, John Wiley \& Sons Ltd, New York 1994 (ISBN : 0-471-00577-0) p. 81.

[28] G. Buyle, Simplified model for the d.c. planar magnetron discharge, PhD thesis, Ghent University 2005 (download is possible from www.draft.ugent.be ).

[29] A.V. Phelps, Z Lj Petrovic, Plasma Sources Sci. Technol. 8 (1999) R21.

[30] H. Hagstrum, Phys. Rev. 96 (1954) 336.

[31] D. Depla, S. Mahieu, R. De Gryse, submitted invited review in Thin Solid Film.

[32] D. Depla, G. Buyle, J. Haemers, R. De Gryse, Surf. Coatings Techn. 200 (2006) 4329-4338.

[33] J. W. Bradley, S. Thompson, Y. Aranda Gonzalvo, Plasma Sources Sci. Technol. 10 (2001) 490-501.

[34] I.V. Svadkovski, D.A. Golosov, S.M. Zavatskly, Vacuum 68 (2003) 283-290.

[35] I. Petrov, F. Adibi, J.E. Greene, W.D. Sproul, W.-D. Munz, J. Vac. Sci. Technol. A 10 (1992) 3283. 
[36] G. Estes, W.D. Westwood, J. Vac. Sci. Technol A 6 (1988) 1845.

[37] A. Furuya, S. Hirono, J. Appl. Phys. 68 (1990) 304.

[38] W.D. Sproul, D.J. Christie, D.C. Carter Thin Solid Films 491 (2005) 1-17.

[39] U. Helmersson, M. Lattemann, J. Bohlmark, A. P. Ehiasarian, J. T. Gudmundsson, Thin Solid Films 513 (2006) 1-24.

[40] S. Berg, T. Nyberg, Thin Solid Films 476 (2005) 215-230.

[41] D. Depla, S. Heirwegh, S. Mahieu, R. De Gryse, Journal of Physics : Applied Physics D, 40 (2007) 19571965.

[42] "Depositing aluminium oxide : a case study of reactive magnetron sputtering", D. Depla, S. Mahieu, R. De Gryse in "Reactive Sputter deposition", D. Depla, S. Mahieu (editors), Springer Berlin Heidelberg New York 2008, ISBN 978-3-540-76662-9.

[43] RSD2008 and SIMTRA can be downloaded from www.draft.ugent.be .

[44] X.Y. Li, D. Depla, W.P. Leroy, J. Haemers, R. De Gryse J. Phys. D: Appl. Phys. 41 (2008) 035203 (6pp) .

[45] H. Poelman, H. Tomaszewski, D. Poelman, D. Depla, R. De Gryse, Surf. Interface Anal. 36 (2004) 1167 1170 .

[46] S. Mahieu, D. Depla, Applied Physics Letters 90 (2007) 121117/1-121117/2.

[47] "Transport of Sputtered Particles Through the Gas Phase", S. Mahieu, K. Van Aeken, D. Depla in "Reactive Sputter deposition”, D. Depla, S. Mahieu (editors), Springer Berlin Heidelberg New York 2008, ISBN 9783-540-76662-9.

[48] K. Van Aeken, S. Mahieu, D. Depla, J. Phys. D.:Appl. Phys. 41 (2008) 205307

[49] E. Martines, R. Cavazzana, G. Serianni, M. Spolaore, L. Tramontin, M. Zuin, V. Antoni Phys. Plasmas 8 (2001) 3042-3050.

[50] I. Petrov, A. Meyers, J.E. Greene, and J.R. Abelson, J. Vac. Sci. Technol. A 12 (1994) 2846

[51] O. Leroy,L. de Poucques, C. Boisse-Laporte, M. Ganciu L. Teulé-Gay, M. Touzeau, J. Vac. Sci. Technol. A 22 (2004) 192-200.

[52] Ekpe S D and Dew S K, J. Vac. Sci. Technol. A 21(2003) 476.

[53] Drüsedau T P, Bock T, John T M, Klabunde F and Eckstein W J. Vac. Sci. Technol. A 17 (1999) 2896.

[54] Wendt R, Ellmer K, Wiesemann K, J. Appl. Phys. 82 (1997) 2115.

[55] Kersten H, Kroesen G M W and Hippler R Thin Solid Films 332 (1998) 282.

[56] Kersten H, Stoffels E, Stoffels W W, Otte M, Csambal C, Deutsch H, Hippler R J. Appl. Phys. 87(2000) 3637.

[57] Hoffman D W J. Vac. Sci. Technol. A 3(1985) 561.

[58] Rossnagel S M J. Vac. Sci. Technol. A 6 (1988) 19.

[59] S. Mahieu, D. Depla, R. De Gryse Surf. Coatings Technol. 202 (2008) 2314-2318.

[60] S. Mahieu, D. Depla, accepted for publication as topical review paper in J. Phys. D : Appl. Phys.

[61] J.A. Thornton, J. Vac. Sci.Technol. 11 (1974) 666-670. .

[62] I. Petrov, P.B. Barna, L. Hultman and J.E. Greene, J. Vac. Sci. Technol. A21 (2003) S117

[63] S. Mahieu, P. Ghekiere, D. Depla, R. De Gryse, Thin Solid Films 515 (2006) 1229-1249. 


\section{Figure Captions}

Figure 1a (left) : Normalized number of publications per year per deposition technique based upon data from the Web of Science.

Figure $1 \mathrm{~b}$ (right) Normalized number of publications per year per deposition technique based upon data from Delphion.

Figure 2. Sputtering yield $\mathrm{Y}$ of $\mathrm{Cu}$ as a function of the energy of $\mathrm{Ar}^{+}$at normal incidence as calculated using the SRIM code. Note that $\left.\mathrm{Y}_{\left(\mathrm{Ar}^{+}\right.}{ }^{+}\right)$is linear over the typical range of operation during magnetron sputtering $\left(\mathrm{E}_{\mathrm{Ar}}^{+}=250-750 \mathrm{eV}\right)$.

Figure 3. The dependence of the sputter yield of several elements (ordered according their position in the periodic table) calculated using SRIM (initial conditions: $300 \mathrm{eV} \mathrm{Ar}$, other input parameters where set at the standard values given by SRIM: lattice binding energy, surface binding energy displacement energy, and normal incidence).

Figure 4. Comparison between the thermal energy distribution for copper evaporated at $1300 \mathrm{~K}$ and the energy distribution of sputtered copper atoms.

Figure 5. The three primary regimes of a gas discharge. The straight line is a typical load line.

Figure 6. Schematic representation of the structure of a glow discharge

Figure 7. Ion energy distributions calculated according to the model of Davis and Vanderslice [24].

Figure 8. Schematic drawing of a post magnetron

Figure 9. Simulated part of a trajectory of an electron on a circular planar magnetron (left) and the resulting torus shaped argon plasma (right).

Figure 10. Two types of planar magnetrons: on the left a circular five $\mathrm{cm}$ diameter magnetron. On the right a rectangular magnetron (notice the clear erosion groove, or race track in both cases).

Figure 11. Magnet configuration for a rotating cylindrical magnetron (left) and a laboratory-scale magnetron of this type.

Figure 12. The inverse of the discharge voltage measured in pure argon discharges as a function of the ion-induced secondary electron yield. Measurements are performed with a conventional $5 \mathrm{~cm}$ diameter circular magnetron (argon pressure 0.3 Pa, discharge current 0.3 A).

Figure 13. The exponent $\mathrm{n}$ (see equation 18) for I-V characteristics measured under different conditions of target thickness and pressure. A conventional $5 \mathrm{~cm}$ diameter circular magnetron was used with a copper target. At low magnetic field (or for thick targets (see $\mathrm{h}=7 \mathrm{~mm}$ ) the slope increases with pressure. However, the opposite behavior is observed for a high magnetic field or thin targets (see $\mathrm{h}=1$ or $\mathrm{h}=2 \mathrm{~mm}$ ).

Figure 14. Some simulated electron trajectories for an unbalanced magnetron (left) together with the cone shape argon plasma. Note that unbalancing the magnetic circuit opens a "leak" in the plasma trap and allows independent control of substrate ion bombardment.

Figure 15. Influence of the magnet balance on the $n$ value (see equation (18)) and the ion-to-atom ratio. The magnet balance, expressed by the value $\mathrm{K}$ (see equation (19)), is changed by shifting the inner or the outer magnet relative to target surface. Several situations are depicted schematically.

Figure 16. Discharge voltage and total pressure behavior during reactive magnetron sputtering of Al in an $\mathrm{Ar} / \mathrm{O}_{2}$ mixture. The argon pressure and the total current were kept constant at $0.3 \mathrm{~Pa}$ and 0.3 A.

Figure 17. Simulation of the reactive sputter deposition process using RSD2008 [43]. The experimental results are shown in figure 14. 
Figure 18. Flow at the transition points as a function of the rotation speed.

Figure 19. By placing a $5 \mathrm{~cm}$ diameter magnetron source in the upper part of a tube, the surface on which material is deposited is well defined, and the influence of the area of this surface and the deposition profile on the hysteresis behavior during reactive magnetron sputtering can be studied. The illustrations shows the result of a simulation in which the deposition profile is included. The oxidation of the deposited target material is shown on the cylinder. Red represents a fully oxidized surface, while blue represents a pure metal surface. Experimental conditions : constant argon pressure $0.3 \mathrm{~Pa}$, constant discharge current $0.43 \mathrm{~A}$, pumping speed , $100 \mathrm{l} / \mathrm{s}$. The tube had a diameter of $26 \mathrm{~cm}$ and a height of $53 \mathrm{~cm}$. The oxygen was introduced at the bottom of the tube. The target material was Al.

Figure 20. Flow chart for SIMTRA describing all steps during the transport of a sputtered particle towards the substrate. $\mathrm{n}_{\mathrm{g}}$ stands for the sputter gas density, $\sigma$ represents the collision cross section, and $v$ the velocity (subscripts s: sputtered atom, g: gas atom, r: relative). $\theta_{\text {com }}$ and $\mathrm{E}_{\text {com }}$ are the polar scattering angle and the kinetic energy in the centre-of-mass system, $p$ represents the impact parameter, $\mathrm{V}(\mathrm{r})$ is the interaction potential, and $\mathrm{R}$ is the distance of closest approach.

Figure 21. Schematic comparison between zone Ic and zone T growth. To indicate the identical normal growth rate of the planes of both grains, an alternating coloring is used. In zone $\mathrm{T}$, an overgrowth of one grain by an adjacent grain is observed. 
Figure 1

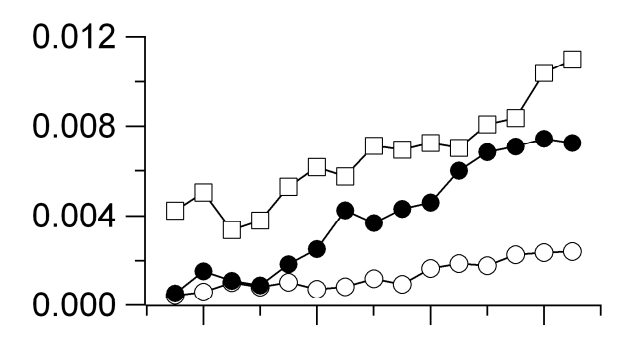

$-\circ$ - electroless

$\longrightarrow-$ sol-gel
$-\square$ electrodeposition
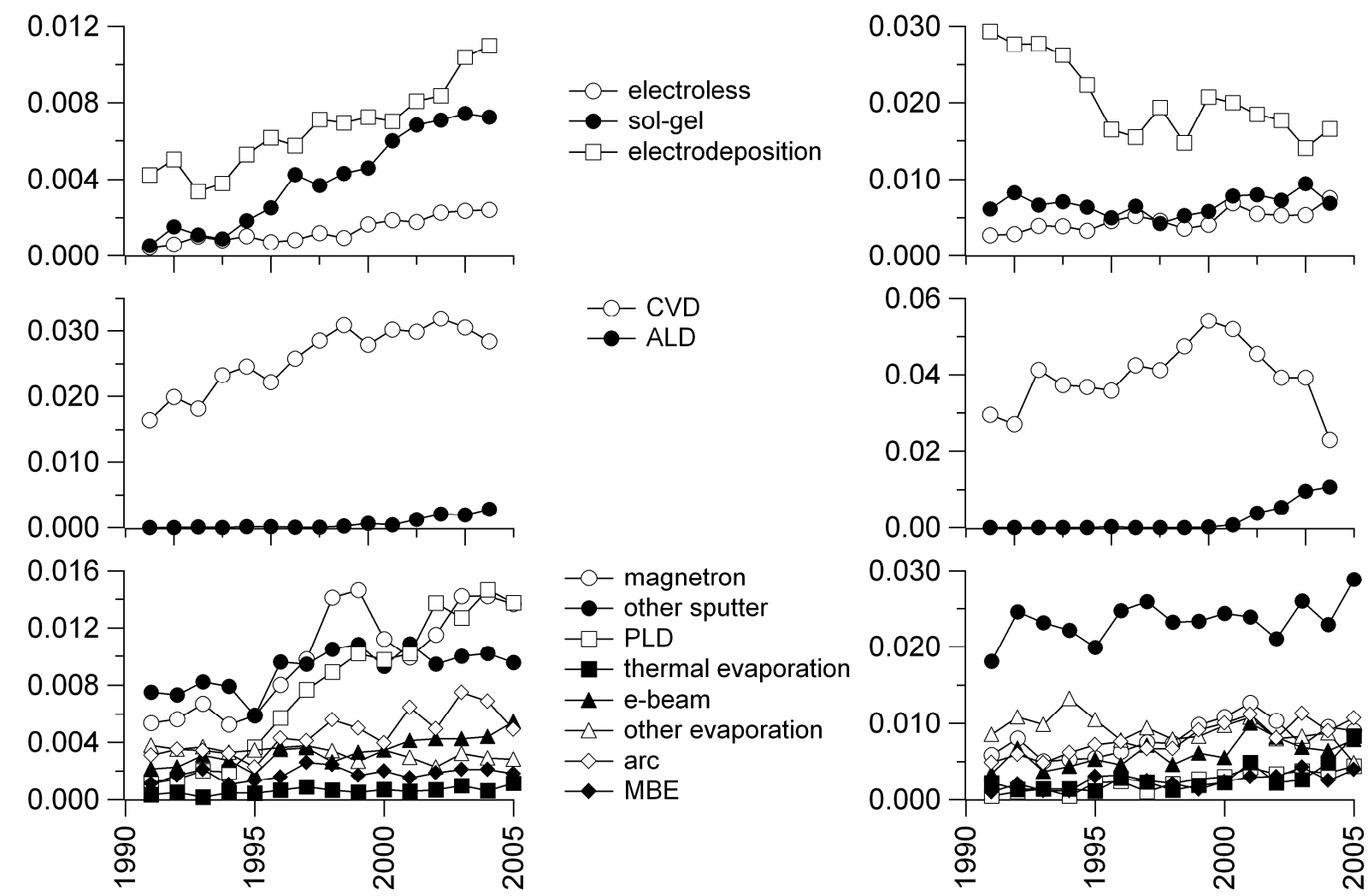

$-\circ$ magnetron

- - other sputter

$-\square-$ PLD

$\rightarrow$ thermal evaporation

$\leftarrow$ e-beam

$\neg$ other evaporation

$\checkmark$ arc

$\neg$ MBE

Figure 2

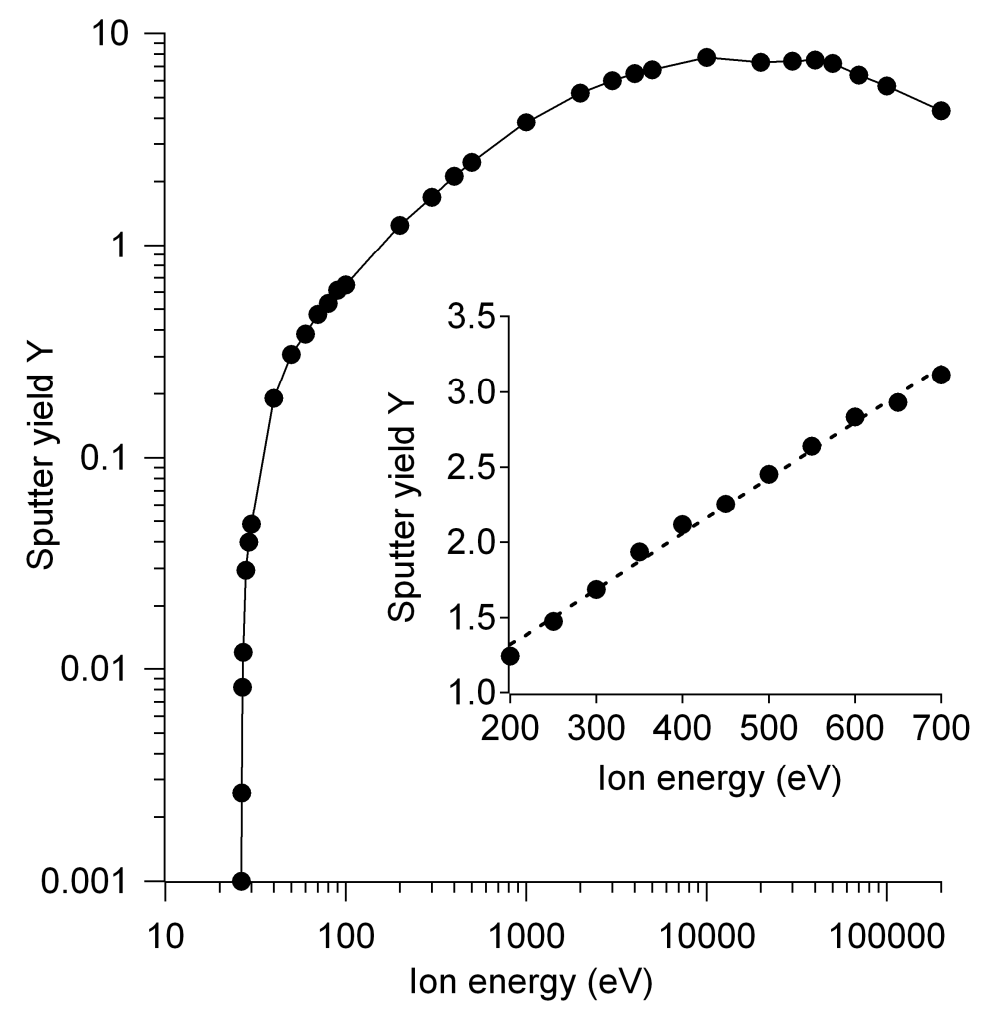


Figure 3

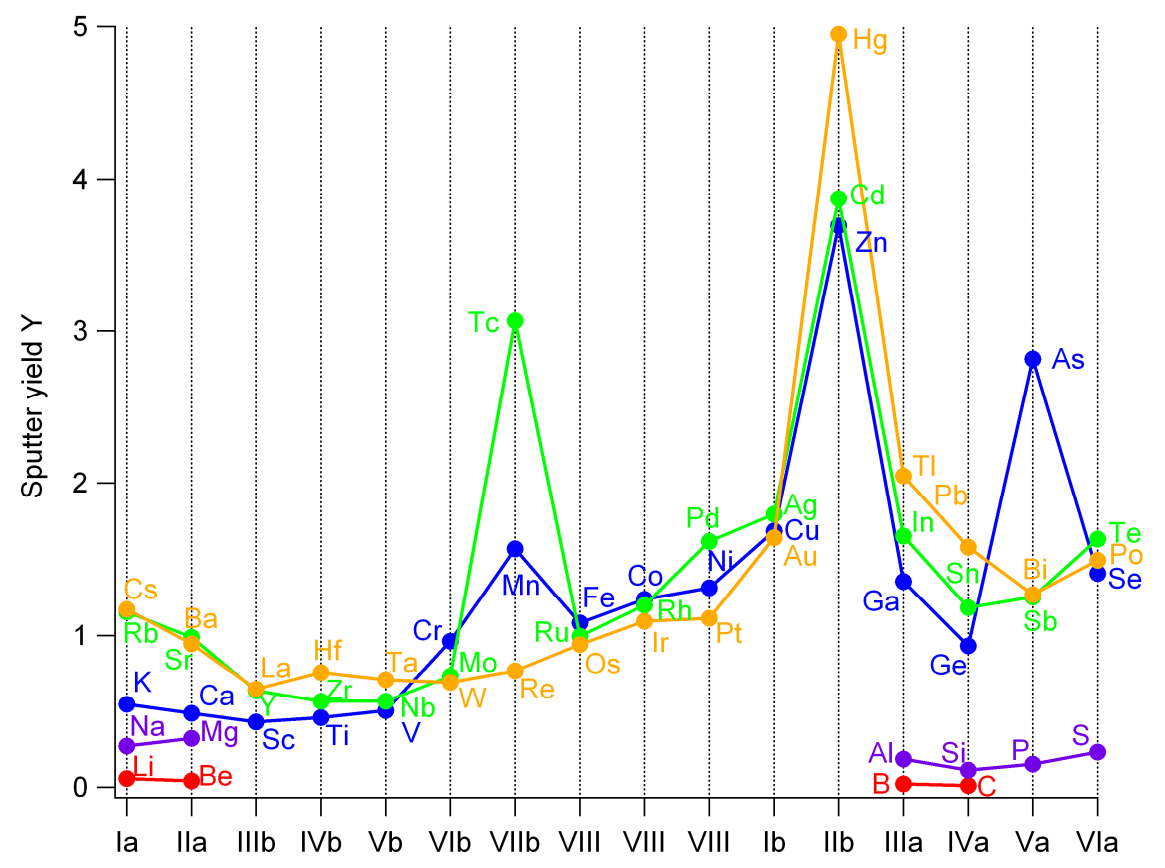

Figure 4

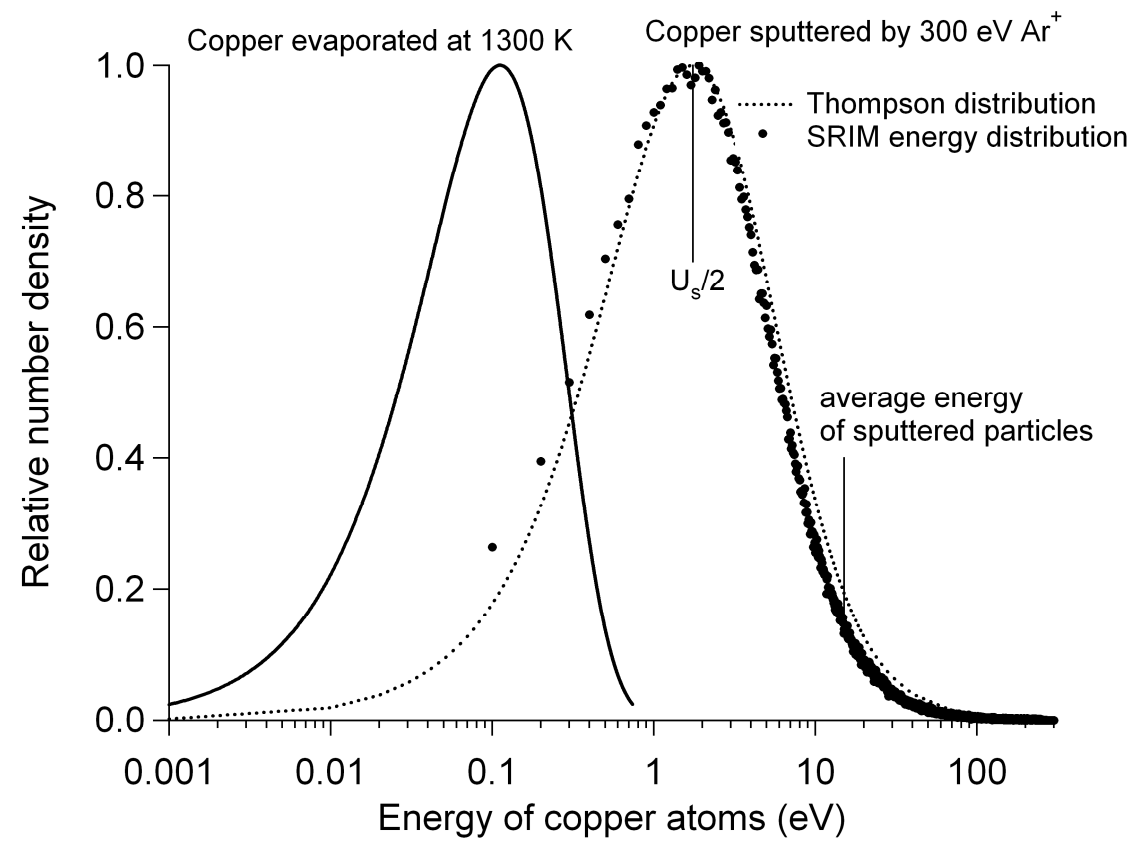


Figure 5

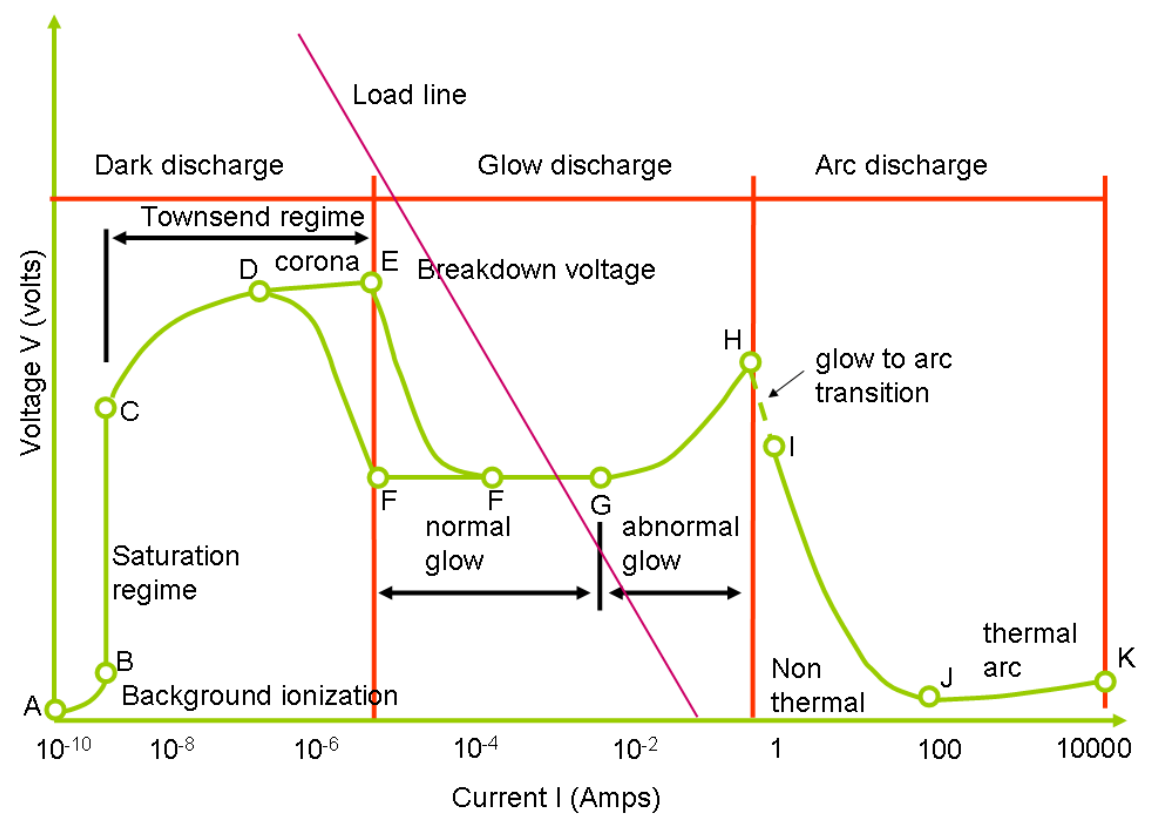

Figure 6

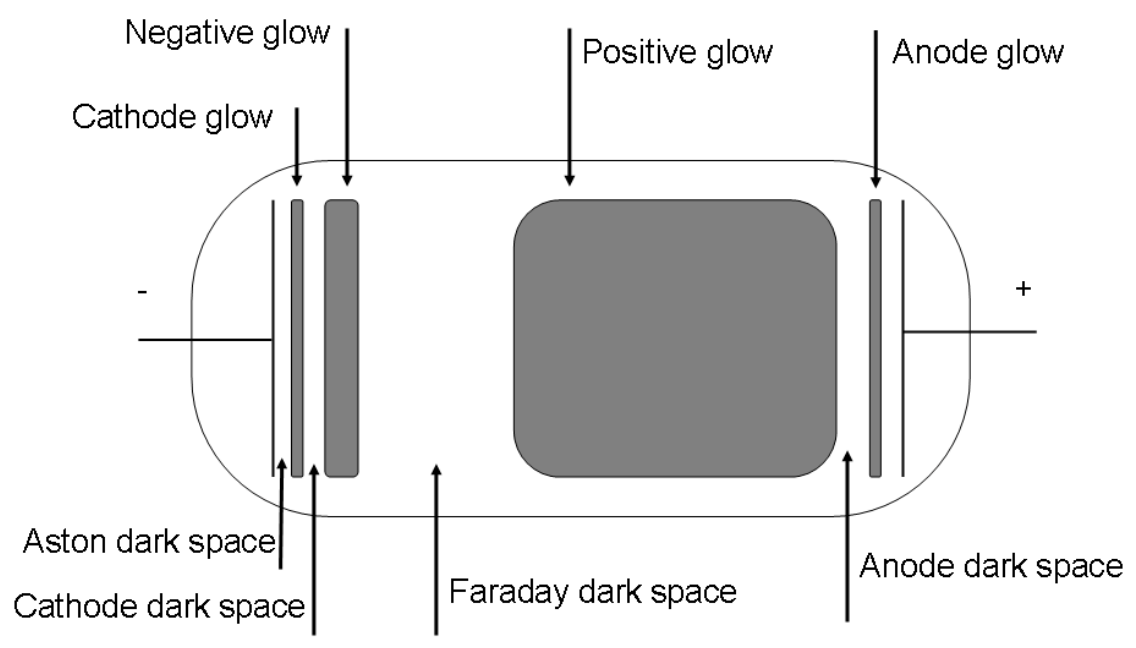


Figure 7

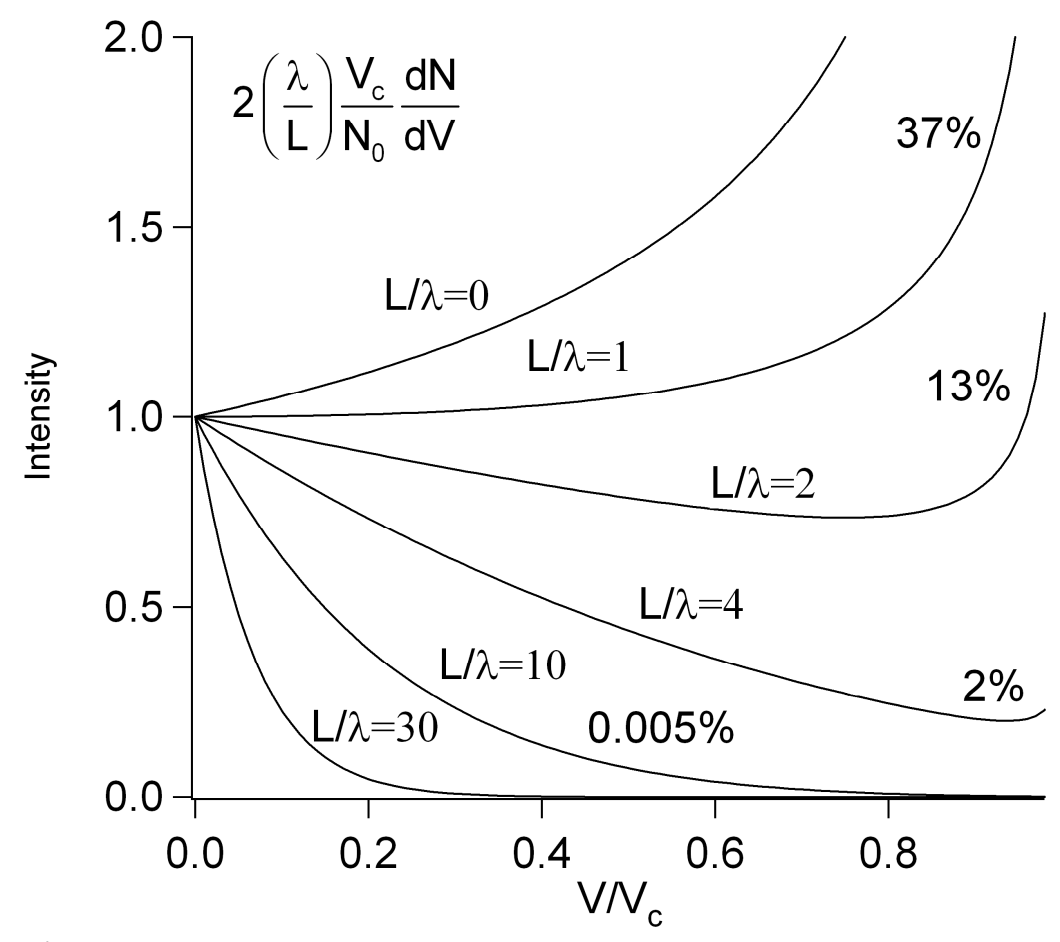

Figure 8

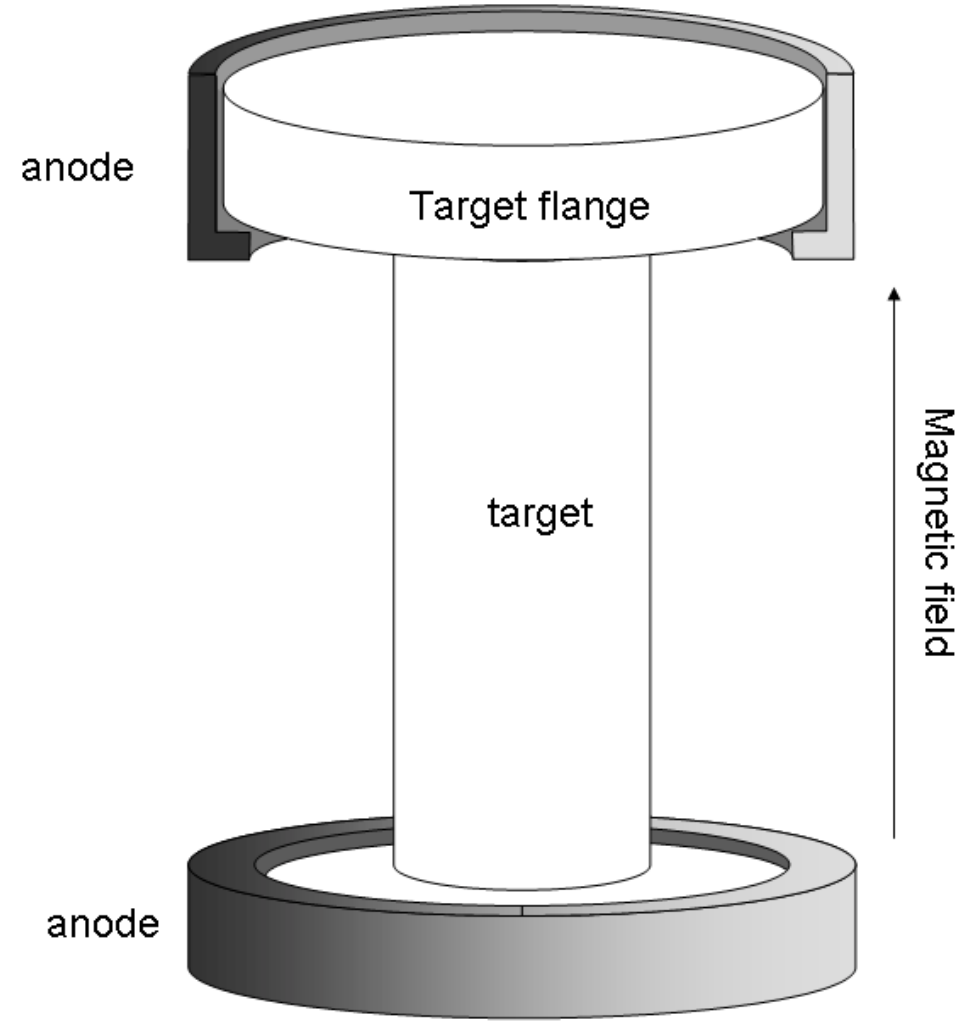


Figure 9
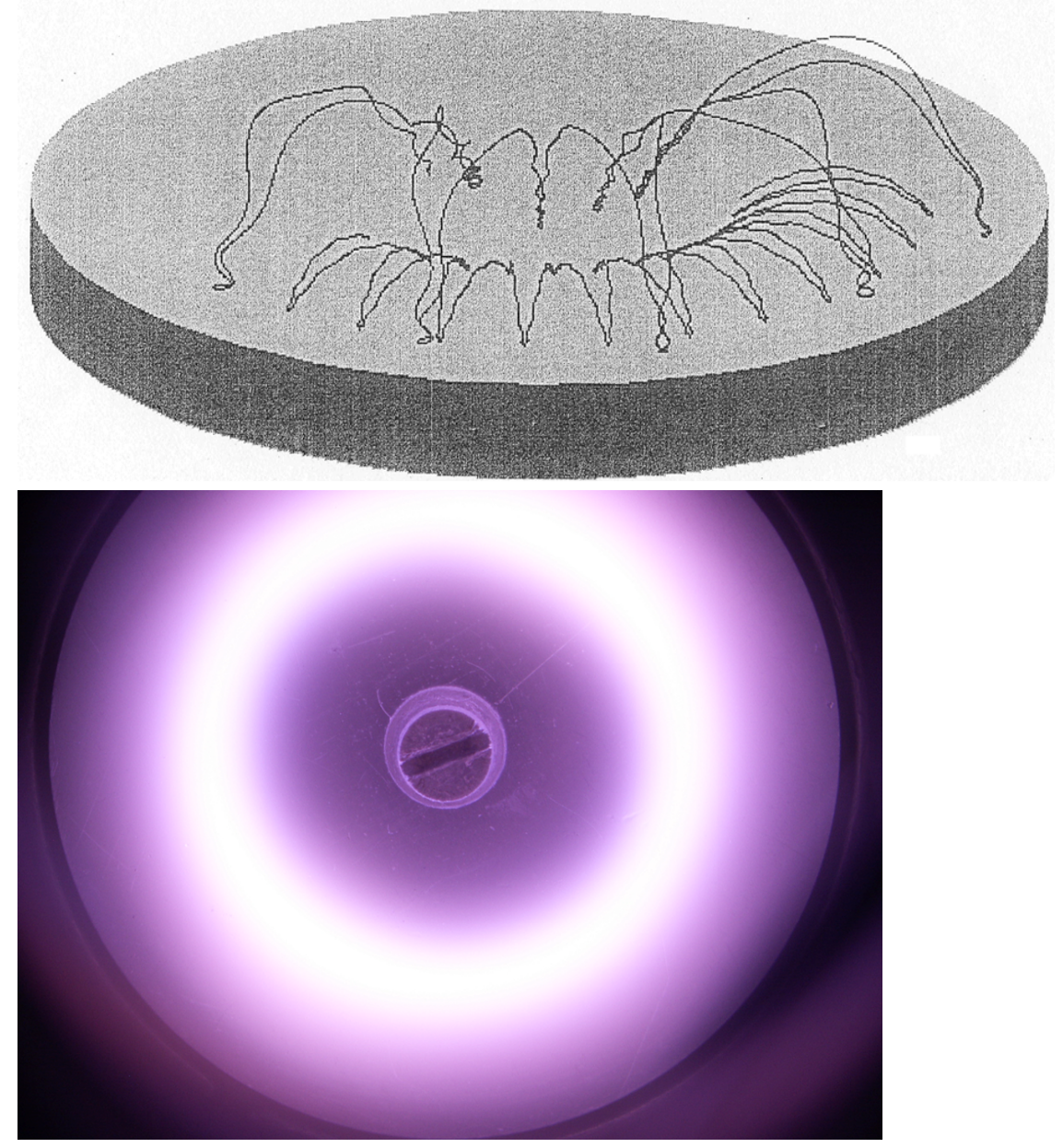

Figure 10

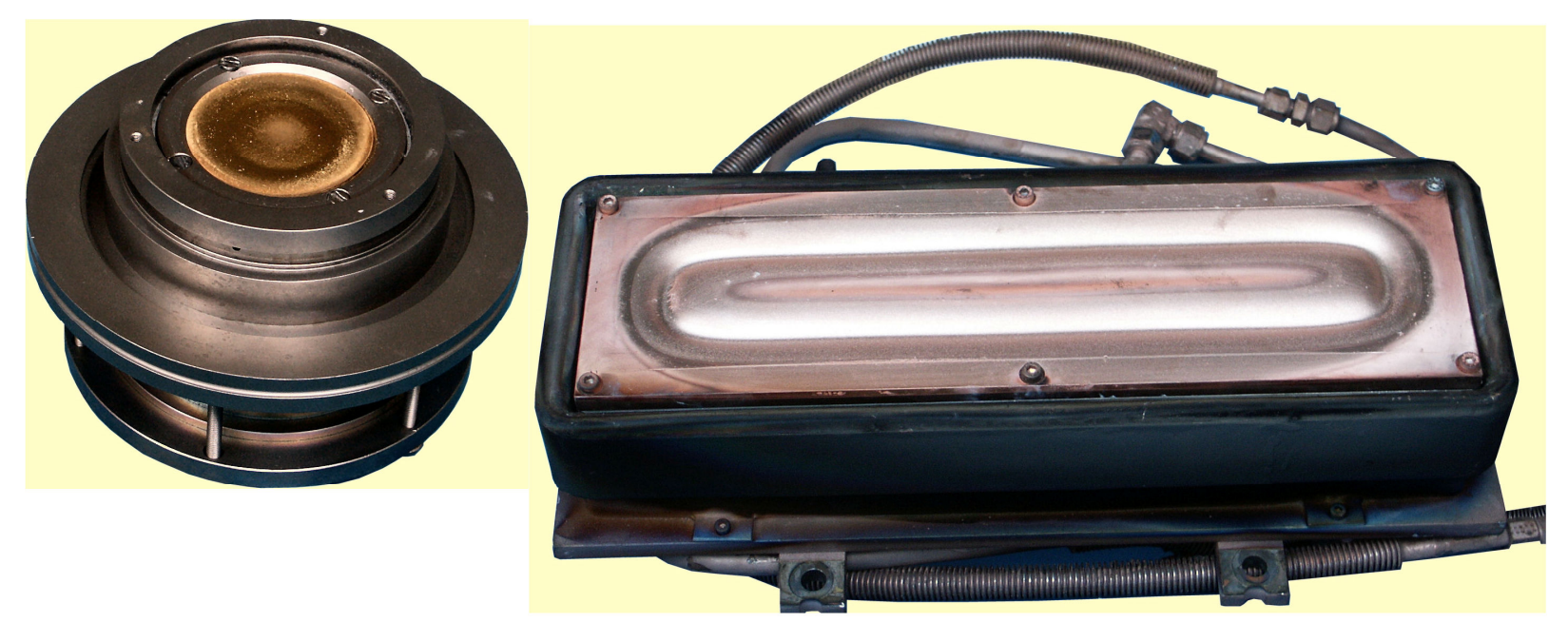


Figure 11

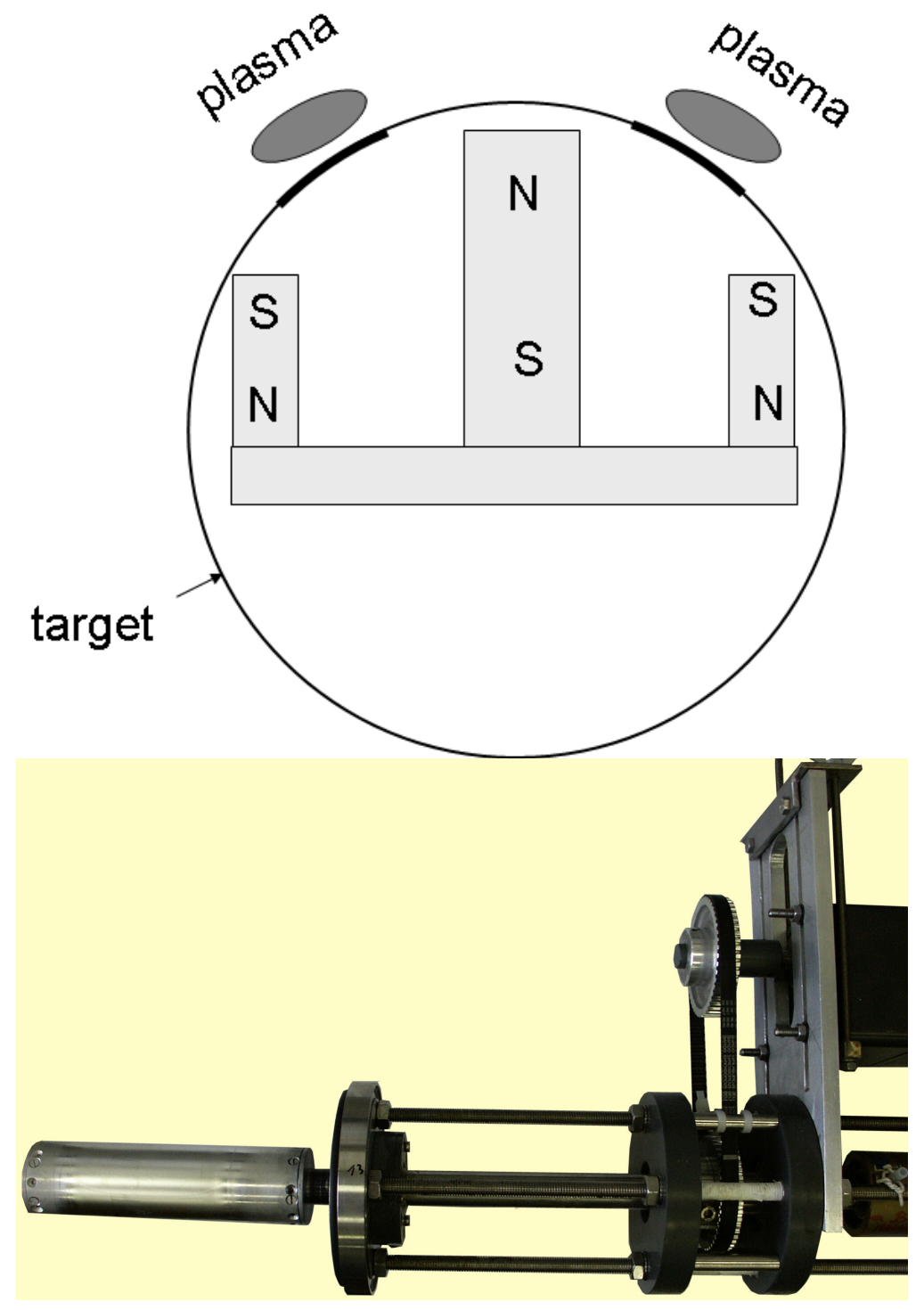


Figure 12

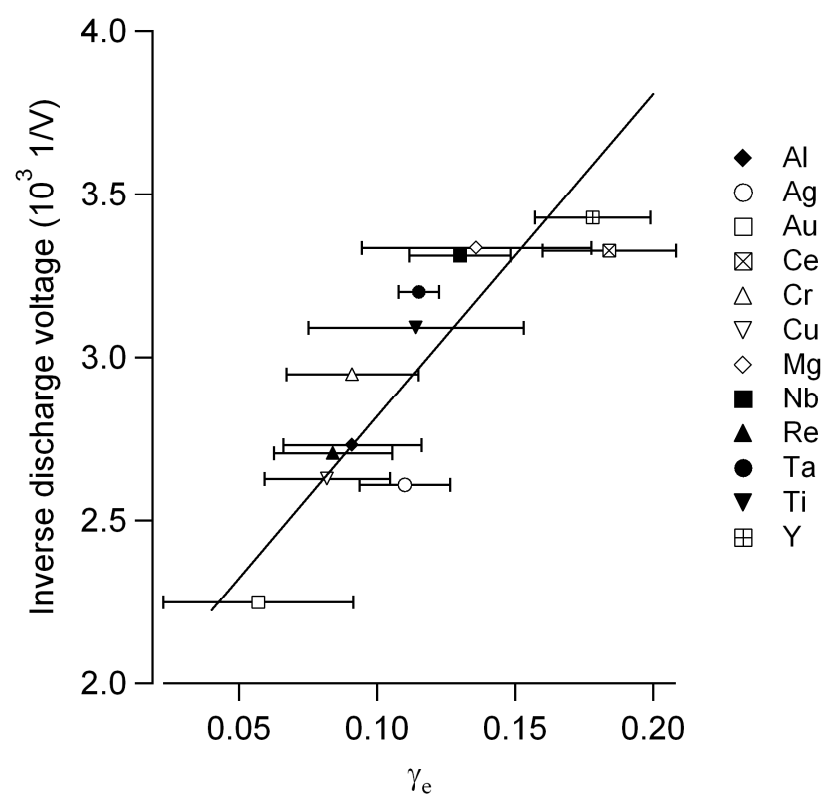

Figure 13

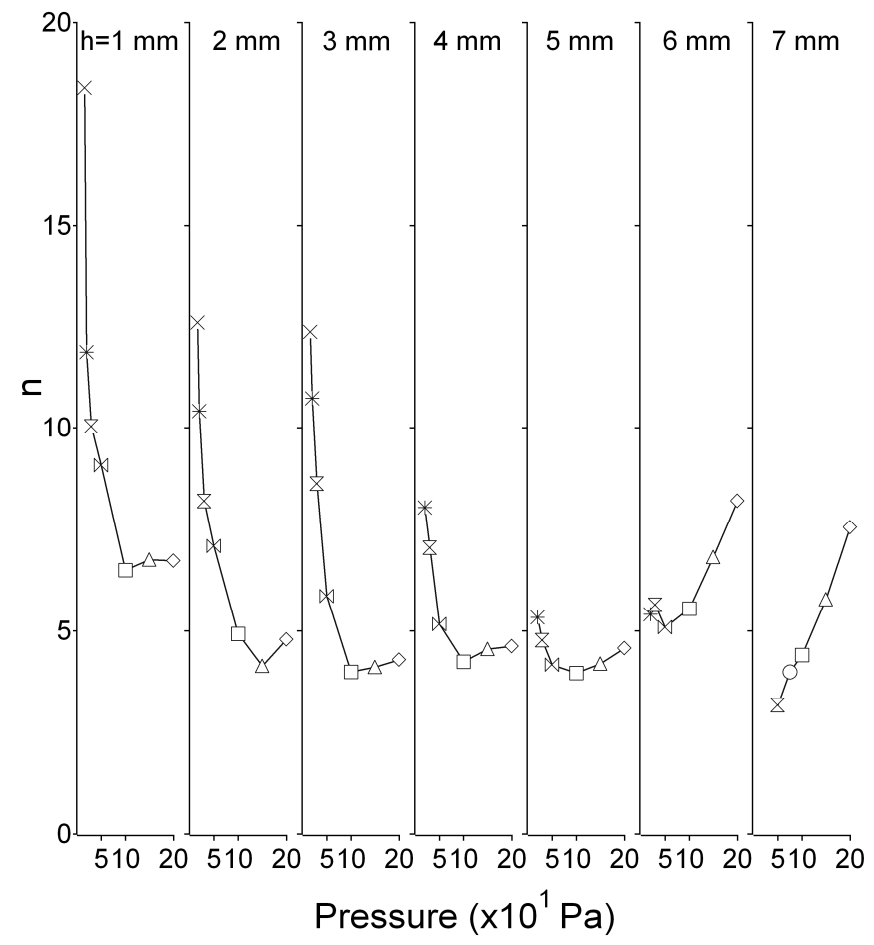


Figure 14
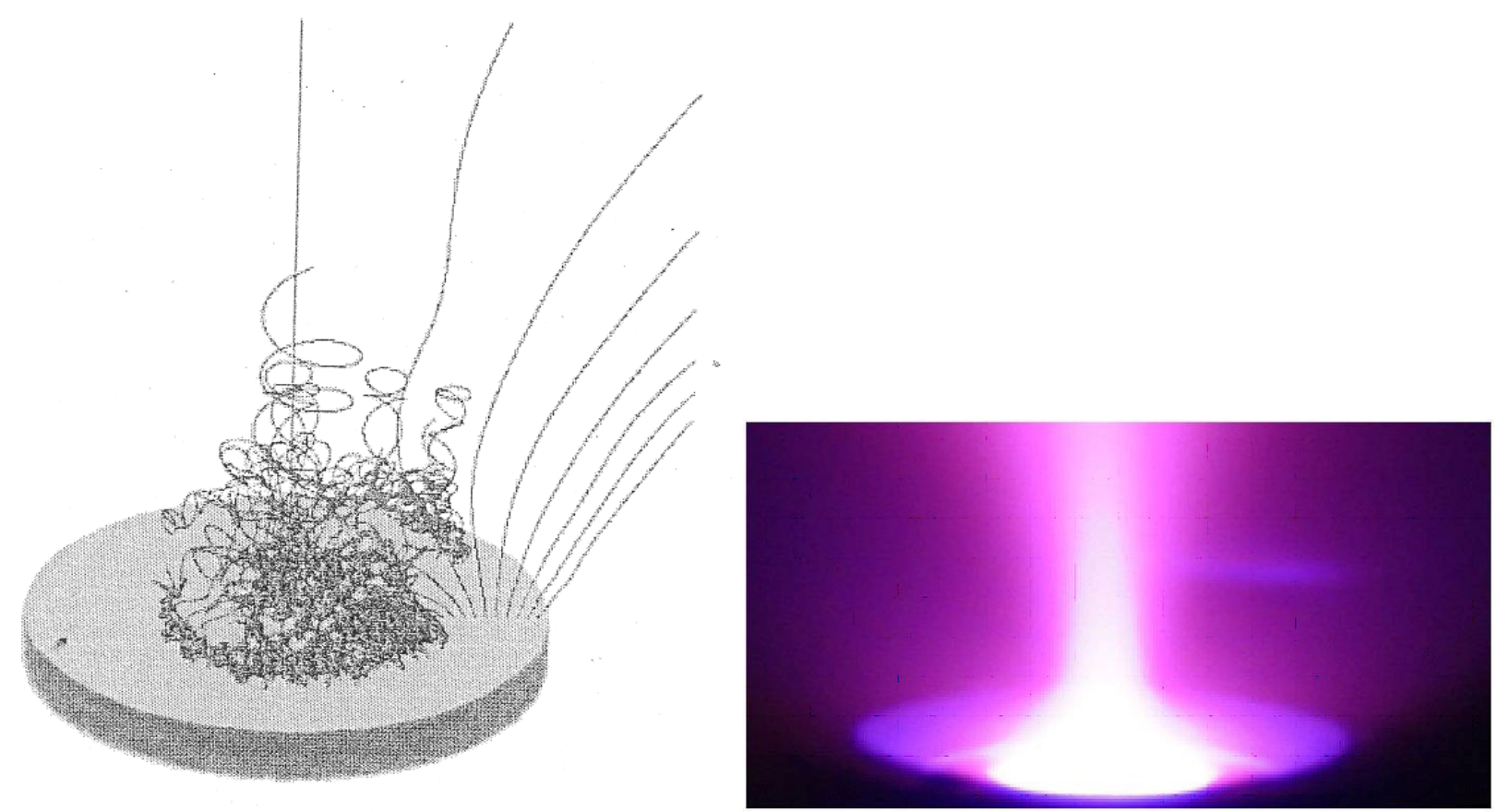

Figure 15

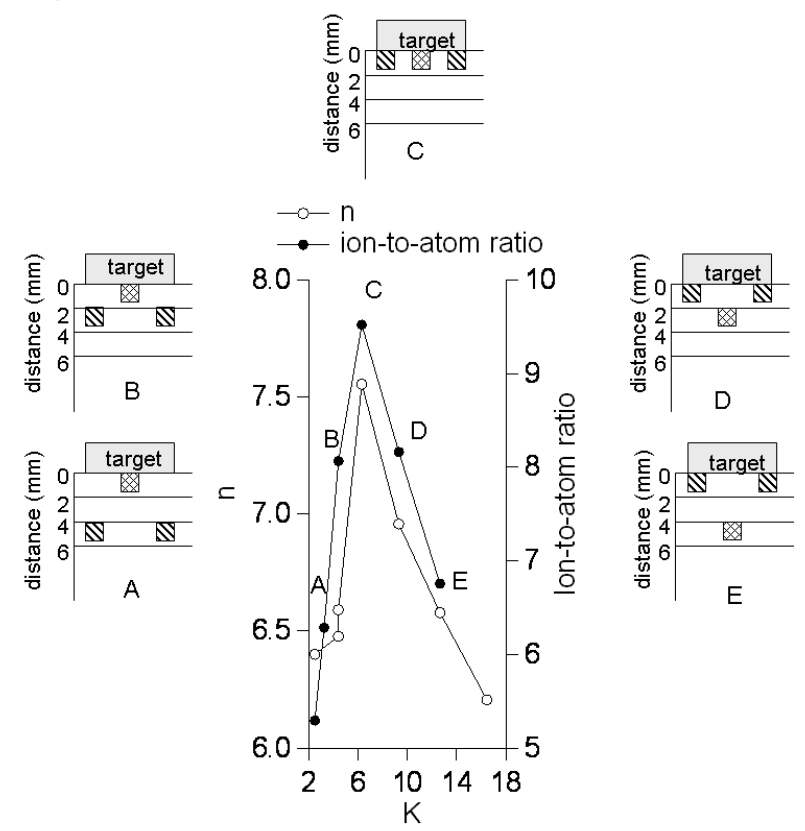


Figure 16

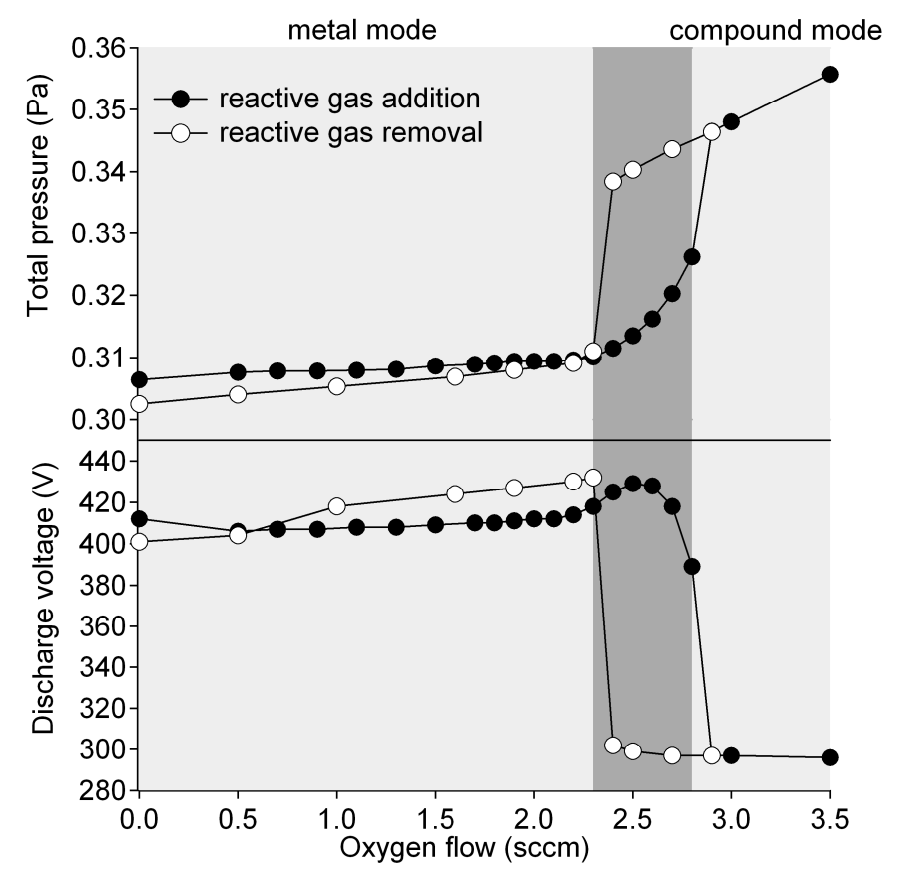

Figure 17

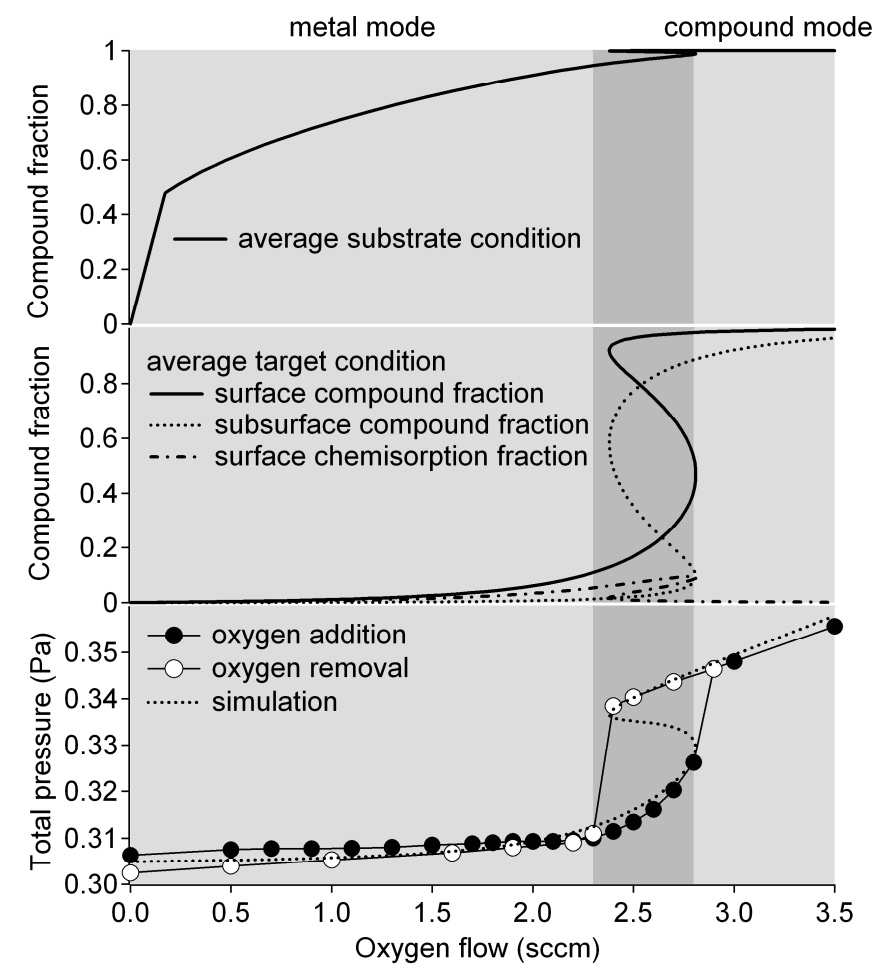


Figure 18

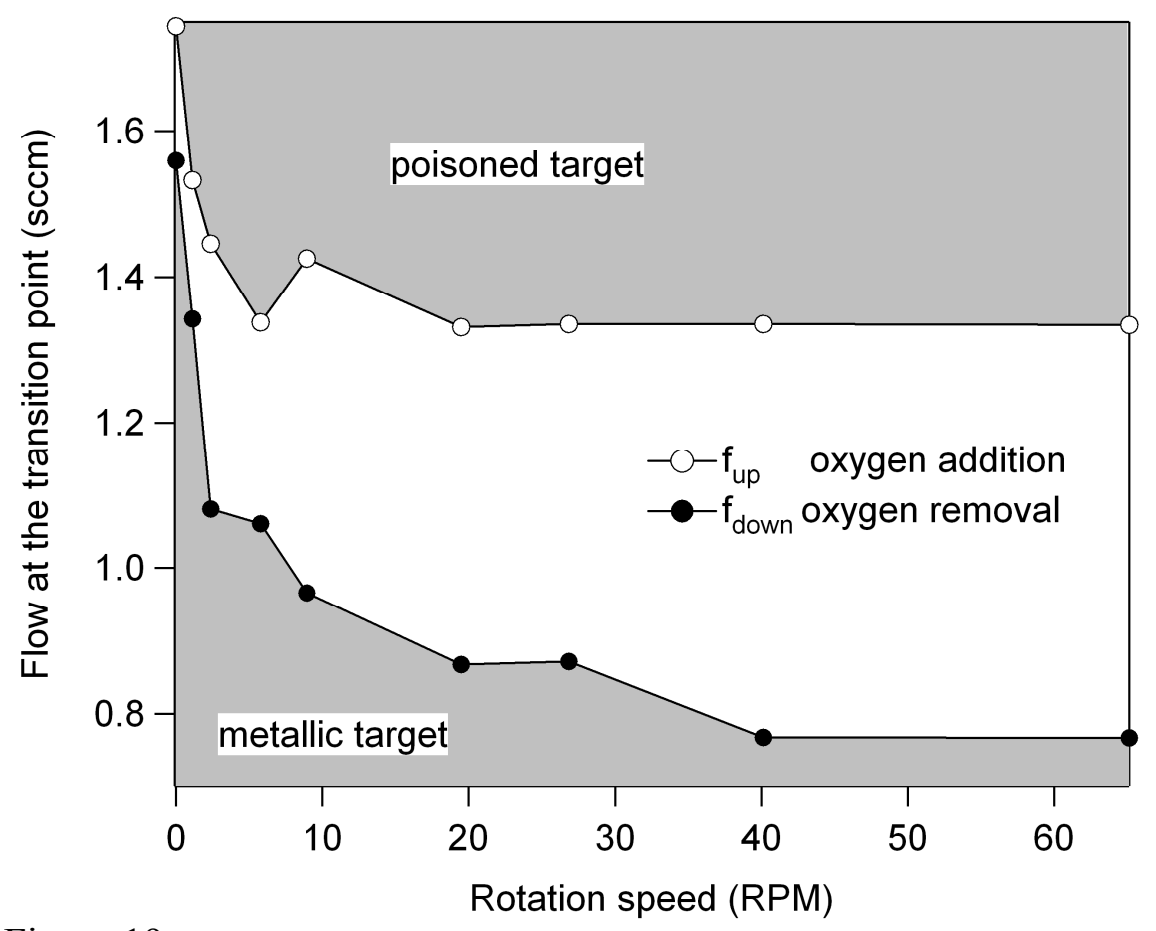

Figure 19

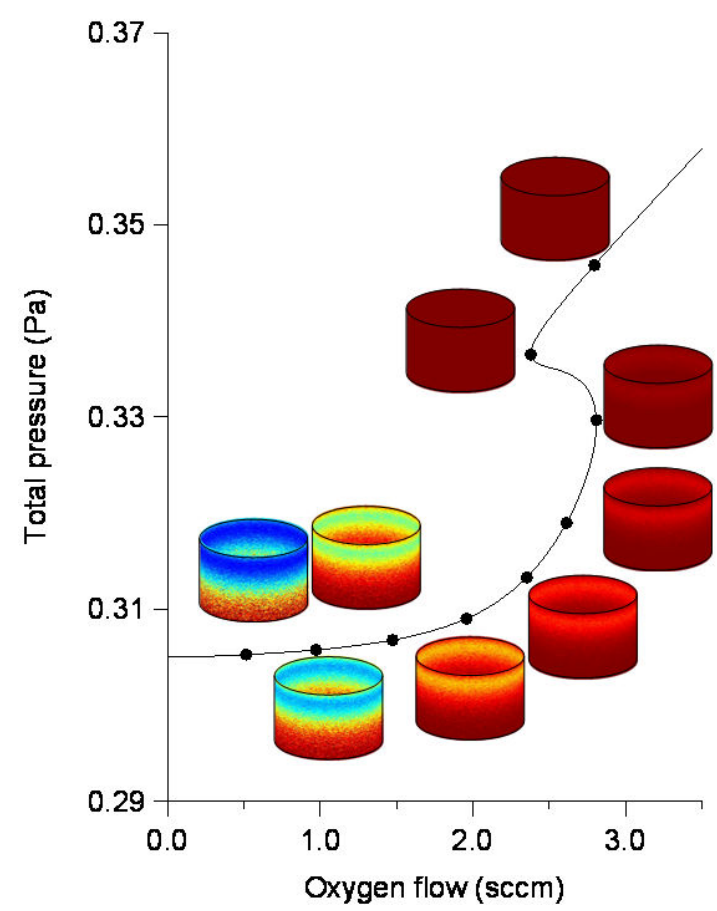


Figure 20

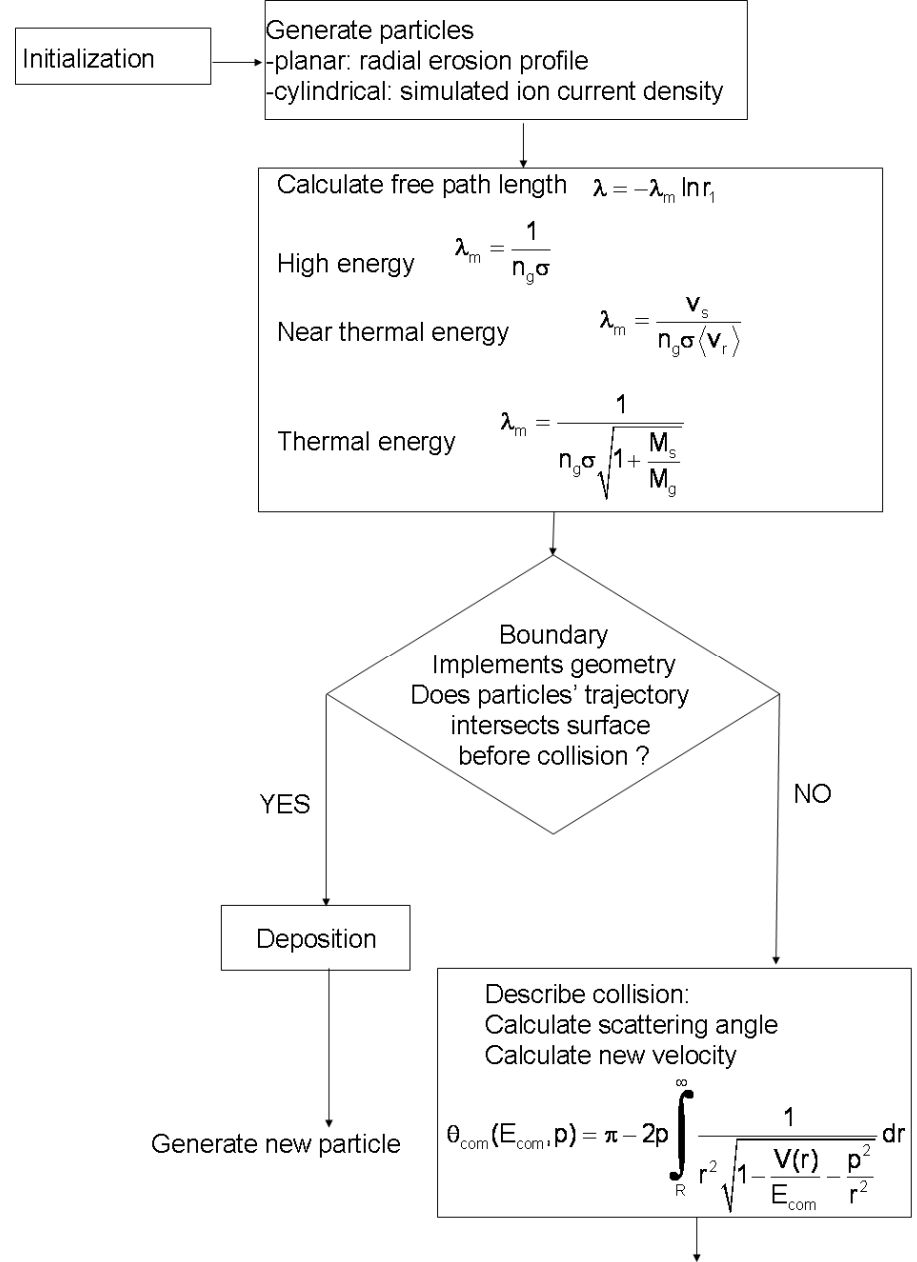

Figure 21

Go back to free path calculation

Zone Ic growth

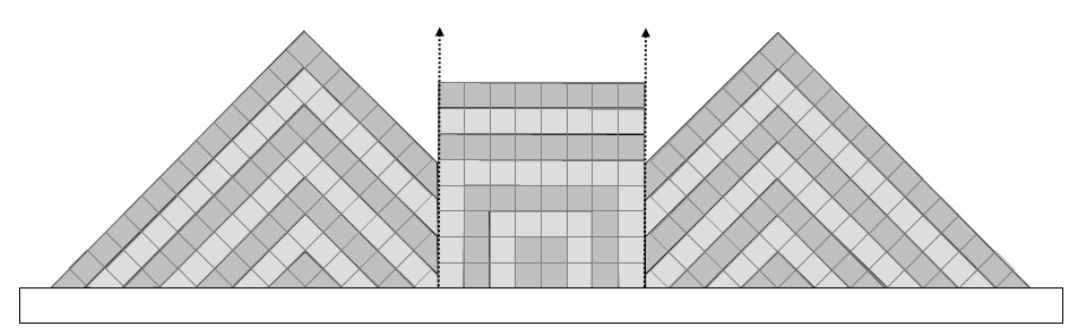

Zone T growth

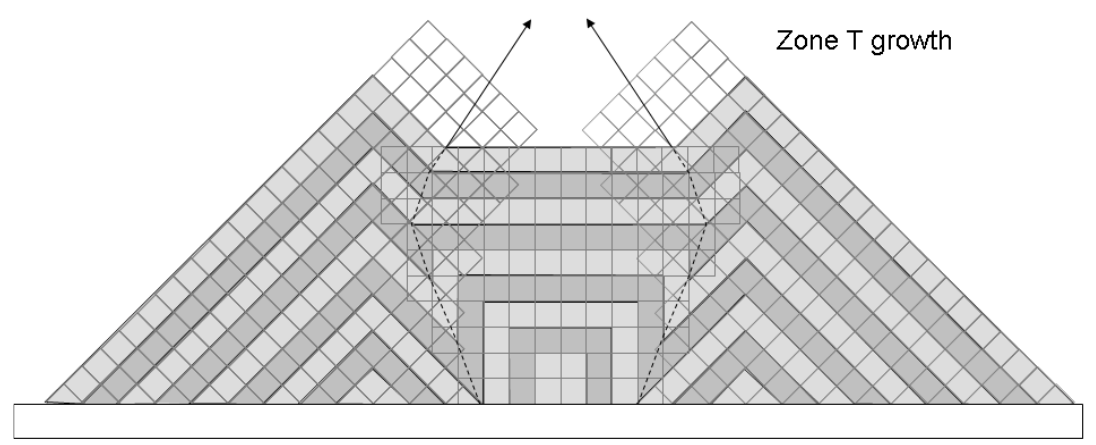

